\title{
The evolution of gravitationally unstable protoplanetary disks: fragmentation and possible giant planet formation
}

\author{
Lucio Mayer ${ }^{1}$, Thomas Quinn ${ }^{2}$, James Wadsley ${ }^{3}, \&$ Joachim Stadel ${ }^{1}$ \\ ${ }^{1}$ Institute of Theoretical Physics, University of Zürich, Winterthurerstrasse 190, 8057, \\ Zurich,Switzerland,lucio@physik.unizh.ch \\ ${ }^{2}$ Department of Astronomy, University of Washington, Seattle, WA 98195, USA, \\ trq@astro.washington.edu \\ ${ }^{3}$ Department of Physics 85 Astronomy, McMaster University, 1280 Main St. West, \\ Hamilton ON L8S4M1 Canada,wadsley@physics.mcmaster.ca
}

\begin{abstract}
We carry out a large set of very high resolution, three dimensional smoothed particle hydrodynamics ( $\mathrm{SPH}$ ) simulations describing the evolution of gravitationally unstable gaseous protoplanetary disks. We consider a broad range of initial disk parameters. Disk masses out to $20 \mathrm{AU}$ range from 0.075 to 0.125 $M_{\odot}$, roughly consistent with the high-end of the mass distribution inferred for disks around T Tauri stars.Minimum outer temperatures range from 30 to 100 $\mathrm{K}$, as expected from studies of the early protosolar nebula and suggested by the modeling of protoplanetary disks spectra. The mass of the central star is also varied although it is usually assumed equal to that of the Sun. Overall the initial disks span minimum $Q$ parameters between 0.8 and 2, with most models being around $\sim 1.4$. The disks are evolved assuming either a locally isothermal equation of state or an adiabatic equation of state with varying $\gamma$. Heating by (artificial) viscosity and shocks is included when the adiabatic equation of state is used. When overdensities above a specific threshold appear as a result of gravitational instability in a locally isothermal calculation, the equation of state is switched to adiabatic to account for the increased optical depth. We show that when a disk has a minimum $Q$ parameter less than 1.4 strong trailing spiral instabilities, typically three or four armed modes, form and grow until fragmentation occurs along the arms after about 5 mean disk orbital times. The resulting clumps contract quickly to densities several orders of magnitude higher than the initial disk density, and the densest of them survive even under adiabatic conditions. These clumps are stable to tidal disruption and merge quickly, leaving 2-3 protoplanets on fairly eccentric orbits (the mean eccentricity being around
\end{abstract}


$0.2)$ after $\sim 10^{3}$ years. Fragmentation is not strongly dependent on whether the disk starts from a marginally unstable state or gradually achieves it; we show that if the disk is allowed to grow in mass from a very light, very stable state over tens of orbital times it still fragments at roughly the same mass and temperature as in the standard disk models. We show that the first stages of the instability, until the appearance of the overdensities, can be understood in terms of the maximum unstable Toomre wavelength and the local Jeans length. A high mass and force resolution are needed to correctly resolve both scales and follow the fragmentation process appropriately. Varying disk mass and temperature affects such physical scales and hence the typical masses of the protoplanets that form. Objects smaller than Saturn or a couple of times bigger than Jupiter can both be produced by fragmentation. Their final masses will then depend on the subsequent interactions and mergers with other clumps and on the accretion of disk material. The accretion rate depends on the disk thermodynamics and is negligible with adiabatic conditions. After $\sim 10^{3}$ years the masses range from just below $1 M_{\text {Jup }}$ to more than $7 M_{\text {Jup }}$, well in agreement with those of detected extrasolar planets.

Subject headings: accretion disks — hydrodynamics — planetary systems:formation — solar system:formation — methods:N-Body simulations

\section{Introduction}

The rapid formation of gas giant planets by gravitational instabilities in a protoplanetary disk (Kuiper 1951;Cameron 1978; Boss 1997) is an appealing alternative to the conventional scenario of accretion of gas onto pre-existing large rocky cores formed by accumulation of planetesimals (Wetherill 1990). The latter scenario seems to require timescales well in excess of disk survival times in dense, highly irradiated environments, like the Orion nebula, where most of the stars in our galaxies are born (Throop et al. 2001), making giant planet formation a rare occurrence. Protoplanetary disks in lower density environments have lifetimes at most marginally consistent with the few millions of years required to form a Jupiter sized planet in the core-accretion scenario at several AU from their star (Pollack et al. 1996, Hubickyj, Bodenheimer \& Lissauer, 2002). However, even in the most favourable scenario it is hard to imagine how planets with masses as large as several Jupiter masses, like many of the observed extrasolar planets (Marcy et al. 2000; Mayor et al. 2001) might also be produced in only a few million years. The problem is not simply that the planet would migrate inward faster than it can accrete enough mass (Nelson \& Papalaloizou 2000; Bate et al. 2003). 
Indeed inward type II migration might be stopped or reversed due to either corotational and Lindblad torques once more realistic disks with profiles that are not simple power laws are considered (Masset \& Papaloizou 2003; Artimowicz \& Peplinski, in preparation), but the mass doubling time of a Jupiter-sized planet after a gap has been opened is of order of $1 \mathrm{Myr}$, even in significantly viscous disks (Artimowicz et al. 1998) - the disk might be dissipated before the planet can grow substantially further. In addition, the evidence on inner rocky cores within the Solar System giants, an inevitable prediction of the core accretion mechanism, is weakening - Jupiter might not have a solid core at all (Guillot 1999a,b). Even for the transiting extrasolar giant HD209458b, where the planetary radius and mass are known (Charbonneau et al. 2000), models of the planet's interior are generally consistent with the absence of a core (e.g. Guillot \& Showman 2002). The overall amount of metals in Jupiter and Saturn is significantly higher than solar, but this does not necessarily reflect the initial metal content of the planets (Boss 1998). In any case, current models of the core-accretion mechanism need a surface density of solids 3-4 times in excess of the minimum solar nebula model (Weidenschilling 1977) for the rocky cores of giant planets to form before 10 million years (Lissauer 1993); if augmented by 99\% times more mass in molecular gas, such a protosolar nebula model will indeed be marginally unstable to gravitational instabilities, which will then become the prevailing formation mechanism because it takes as little as a thousand years (Boss 1997,2000, 2001). On the other hand, gravitational instabilities in a protoplanetary disk are hard to treat correctly due to both various numerical pitfalls that can arise in the simulations and the difficulty of accounting properly for all the cooling and heating mechanisms present in real protoplanetary disks (Pickett et al. 1998, 2000 a,b, 2003). Due to the complexity of the problem, the natural first step is to adopt a simple thermodynamical description of the disk and use a very high resolution to probe in great detail the highly nonlinear dynamics associated with gravitational instability. In Mayer et al. (2002) we showed that, under certain conditions, a system of gas giants can arise whose properties are reminescent of those of known extrasolar planetary systems. Essential to this result was the ability to achieve very high spatial and mass resolution thanks to the fast parallel Tree+SPH code GASOLINE (Wadsley, Stadel \& Quinn 2003). Here we describe the results of a much larger suite of simulations, exploring a wide parameter space in terms of both disk structural properties and thermodynamics, as well as addressing in detail the various numerical aspects of the calculations and how these can affect the final outcome of the gravitational instability. We will also discuss the reliability of the initial conditions used in the simulations and the structural properties of the protoplanets formed in some of the runs. In a forthcoming paper we will discuss the effects of both irreversible heating and radiative cooling in high resolution disk simulations. 


\section{Initial Conditions}

The self-gravitating disk models are a collection of 200,000 or 1 million SPH particles rotating around a central star. Disks have masses ranging between 0.075 and $0.125 M_{\odot}$, comparable to the most massive among T Tauri disks (Beckwith et al. 1990; Dutrey et al. 1996) and 3-4 times more massive than the minimum mass solar nebula (Weidenschilling 1977). However, these masses are still lower than those expected in the early stages of the formation of the star-disk system from the infalling molecular cloud material, as suggested by hydrodynamical simulations (Pickett et al. 1998, 2000a). Initially disks extend from 4 to $20 \mathrm{AU}$ and have a surface density profile $\Sigma(r) \sim r^{-1.5}$ with an exponential cut-off at both the inner and outer edge. We do not apply any boundary conditions, a difference with most previous works on the subject (e.g. Boss 1998, 2002; Nelson et al. 1998, 2000), so disks are free to expand and, at the same time, material can accrete onto the central star due to transfer of angular momentum resulting from artificial viscosity and eventual non-axisymmetric instabilities. The central star is a softened point mass; its typical mass, $M_{s}$, is equal to $1 M_{\odot}$ (see Table 1 ), and it can wobble in response to the time-dependent disk potential; its softening is $2 \mathrm{AU}$, so that the innermost annulus of material effectively feels a point-mass potential — we use a spline kernel softening (Hernquist \& Katz 1989) for both the central star and gas particles, hence the potential is keplerian at two softening scale lengths. The keplerian velocity of gas particles is corrected to account for the effect of pressure (gas particles thus move at slightly sub-keplerian speeds). The initial vertical density structure of the disks is imposed by the hydrostatic equilibrium for an assumed temperature profile $T(r)$. The disks are not seeded with non-axisymmetric perturbations as normally done in grid-based simulations (e.g. Boss 1998) — Poisson noise is indeed present in an SPH simulation even at fairly high resolution (at a level of $0.1 \%$ in our 1 million particles runs, comparable to the seeds in Boss' models) due to the discrete representation of the system.

The softening of gas particles, $\varepsilon_{s}$, is constant with time in our runs, while in some of the previous SPH simulations of the disk instability it was evolving (typically decreasing) as the smoothing length (e.g. Nelson et al. 1998, 2000). Our implementation is preferred in most astrophysical problems as it avoids the unphysical situation of having particles with varying gravitational potential energy. A good choice for the softening is one that allows a gravitational force resolution initially close to the resolution of pressure forces, hence $\varepsilon_{s} \sim h$, where $h$ is the SPH smoothing length calculated over 32 neighbors (Bate \& Burkert 1997). In the latter case the softening scales with the mass of the gas particles, $m_{g}$, as $m_{g}^{1 / 3}$, because so does the SPH smoothing length; as a result, we will use smaller softenings in simulations with higher mass resolution. The latter type of scaling between softening and mass is widely adopted and has been repeatedly shown to be reliable in hydrodynamical simulations of 
structure formation (e.g. Hernquist \& Katz 1989; Thacker et al. 2000). As explained in Bate \& Burkert (1997), however, the gravitational softening must be small enough in order to resolve the local Jeans mass even in high density regimes if collapse or fragmentation is the aim of our calculation. The Jeans mass is bigger than the entire system in our disks initially, but it will eventually decrease if the local density increases due to gravitational instabilities, and the SPH smoothing length will also decrease correspondingly. Therefore, in order to leave room for better force resolution in the later stages of the simulations we choose $\varepsilon_{s} \sim 0.5 h$ (where $h$ is the initial smoothing length) in the majority of our simulations. However, in a few runs we explored the effect of choosing a bigger or smaller softening, and the results will be discussed in section 3.3. Gravitational forces between distant particles are calculated using the hierarchical tree method with opening angle parameter $\theta=0.7$ (Barnes \& Hut 1986). In particular, GASOLINE uses a binary tree and multipole expansions are carried out up to the hexadecapole order (see Stadel 2001 for details). The code uses multistepping to advance particles in an efficient way with a leapfrog integrator (see Wadsley, Stadel \& Quinn 2003).

In most of the simulations the disk is initialized with a mass and temperature profile so as to obtain a desired minimum Toomre $Q$ parameter in the outer, colder part of the disk, similar to what was done in Boss $(2001,2002 \mathrm{a}, \mathrm{b})$. We recall that $Q=\Omega v_{s} / \pi G \Sigma$ for a thin disk in keplerian rotation (Toomre 1964), where $\Omega$ is the angular velocity, $\Sigma$ is the disk surface density, $v_{s}$ is the sound speed (which is proportional to $\sqrt{T}$, where $T$ is the temperature) and $G$ is the gravitational constant. Figure 1 shows examples of Q profiles in some of our models. We also performed a run in which the disk approaches a given minimum Toomre parameter, $Q_{\text {min }}$, from a very stable state (high $Q$ ), starting from a very low initial mass (run DISLgr in Table 1) which is grwon over time by an order of magnitude. The latter simulation allows us to test the dependence of our results on the way the standard initial conditions are set up. The shape of the temperature profile is the same for each model (Figure 2) and is similar to that used by Boss (1998,2001); temperature depends only on radius, thus there is no difference between midplane and an atmosphere. Between 5 and 10 AU the temperature goes $\sim r^{-1 / 2}$, which resembles the slope obtained if viscous accretion onto the central star is the key driver of disk evolution (Boss 1993). Between 4 and 5 AU the temperature profile rises more steeply, being partially determined by irradiation from the central stellar source in agreement with the $3 \mathrm{D}$ radiative transfer calculations of Boss (1996), while it smoothly flattens out for $R>10 \mathrm{AU}$ and reaches a constant minimum temperature (an exponential cut-off is used). The minimum temperature ranges between 35 and $100 \mathrm{~K}$ (typically is around $50 \mathrm{~K}$ ); it is implicitly assumed that the disk temperature is related to the temperature of the embedding molecular cloud core from which the disk would be accreting material (Boss 1996). Note that, at least for the protosolar nebula, $50 \mathrm{~K}$ 
is probably a conservative upper limit for the characteristic temperature at $R>10$ AU based on the chemical composition of comets in the Solar System (temperatures as low as $20 \mathrm{~K}$ are suggested in the recent study by Kawakita et al. 2001). Outer temperatures between 30 and $70 \mathrm{~K}$ are found also for several $\mathrm{T}$ Tauri disks by modeling their spectral energy distribution assuming a mixture of gas and dust and including radiative transfer (D'Alessio et al. 2001).

In addition to the $Q$ parameter, another important measure of the susceptibility of a disk to gravitational instabilities is provided by the $X_{m}$ parameter, $X_{m}=\Omega^{2} R / 2 m \pi G \Sigma, m$ being the order of the unstable mode and $R$ being the disk radius. An extensive literature of numerical experiments conducted for both collisionless and gaseous disks has shown that $X_{m}$, coupled with $Q$, provides a good measure of the susceptibility of the disk to swing amplification of a given mode. In swing amplification a leading wave is amplified into a higher amplitude trailing wave, and if the latter can be turned back into a leading wave a feedback loop is initiated that can produce a disturbance whose magnitude is orders of magnitude greater than that of the initial wave (Binney \& Tremaine 1987). In Figures 1 and 3 we show the $Q$ profile and $X_{m}$ profiles $(m=2,3)$ for two of our disk models; other models (see Table 1) differ only in the value of $T$ or $\Sigma$ and hence their profiles can be easily recalculated (they differ only in the normalization, not in the shape). Strong swing amplification typically requires $X_{m}<3$ and $Q<3$ in some region of the disk (Binney \& Tremaine 1987); both conditions are marginally satisfied only at the very edge of the disk for $m=2,3,4$ in most of our models (e.g. DISL1 and DISH1, see Figure 3), while they are definitely satisfied in model DISL4 at $R>15 \mathrm{AU}$ (see Table 1; this model has a central star whose mass, $M_{s}$, is half of the standard value, and as $\Omega^{2} \sim M_{s}, X_{m} \sim M_{s}$, hence $X_{m}$ is a factor of 2 lower for any $m$ compared to other disk models having the same mass). In section 3.1.1 we will discuss the role of that the initially sharp outer edge of the disks might have in swing amplification.

We performed both locally isothermal (ISO) and adiabatic (ADI) runs. The gas is always assumed to be purely in the form of molecular hydrogen, hence we assume $\mu=2$ for the molecular weight. In what we call the adiabatic runs we solve a thermal energy equation which also includes heating from artificial viscosity (see below), in particular the quadratic term in the latter accounts for irreversible shock heating (Monaghan \& Gingold 1983). The equation of state has the form $P=(\gamma-1) \rho u$, where $P$ is the pressure, $\rho$ is the density and $u$ is the specific internal energy of the gas and $\gamma$ is the ratio between the specific heats. We assume $\gamma=1.4$ in most of the simulations(appropriate for molecular hydrogen with rotovibrational transitions) but, more in general, we vary it in the range $1-1.4$. The locally isothermal equation of state stands on the assumption that any heating is instantaneously radiated away. The latter is simply written as $P=\rho u$, where $u$ is now constant (therefore no thermal energy equation is solved in this case) and is given by $u=k_{B} T / \mu$, where $T$ is 
the temperature and $k_{B}$ is the Boltzmann constant. In our simulations the gas is isothermal in a Lagrangian sense, i.e. the thermal energy of a given particle is assigned based on its initial distance from the star and does not vary, irrespective of its subsequent motion through the disk. We note that, although some radial mixing is expected to occur because of developing non-axisymmetric instabilities, the initial temperature of the disk is constant by construction throughout most of the outer disk, where the strongest instabilities should develop; we verified that radial motions of particles do not have any significant impact on the outer disk temperature profile, thereby the hypothesis of local isothermality is self-consistent in the regions of interest (Figure 2). Values of $\gamma$ smaller than the canonical 1.4 yield smaller pressure and are expected to produce results closer to those of the locally isothermal runs.

All our simulations include artificial viscosity in the standard Monaghan formulation (Monaghan \& Gingold 1983) plus the Balsara correction term (Balsara 1995) to reduce unwanted viscosity in purely shearing flows (see Wadsley, Stadel \& Quinn 2003 for details). Artificial viscosity appears in both the momentum and the thermal energy equation. In SPH codes artificial viscosity is introduced for a number of reasons, primarily to avoid particle interpenetration and reduce post-shock oscillations in high Mach number flows. The magnitude of the viscosity terms becomes smaller with decreasing smoothing length, hence with increasing resolution. In most of the runs the linear and quadratic coefficients of artificial viscosity are set to, respectively, $\alpha=1$ and $\beta=2$. Although this choice is standard in three-dimensional SPH calculations (see for example Hernquist \& Katz 1989, Navarro \& Benz 1991, Thacker et al. 2000), it is still borne out of classic tests like the shock tube and the isothermally collapsing gas cloud, and it is not guaranteed to be optimal in more complex systems like those considered here. In particular, whereas the quadratic term is needed to properly follow the shocks that will eventually develop during the gravitational instability, the linear term mainly damps the velocities of particles, reducing the noise inherent to the SPH technique. One worry is that numerical viscosity, by acting as an effective pressure, might smear out even physical small-scale features in the velocity field (for example in a region that is about to collapse due to gravitational instability) and generate spurious angular momentum transfers (Thacker et al. 2000). Heat generated by artificial viscosity when the thermal energy equation is solved might also affect the disk evolution. We investigate how artificial viscosity affects our results in both isothermal and adiabatic calculations by varying the value of the $\alpha$ and $\beta$ coefficients (see Table 1 and section 3.4). 


\section{Results}

In what follows we describe the results of our large suite of simulations. The various setups are indicated in Table 1. In the same Table we also indicate whether clumps (protoplanets) are formed or not in a given run. Disks are typically followed for about 12-15 orbital times, where we define the characteristic orbital time as that at $R=10 \mathrm{AU}$ (halfway

between the center and the edge of the disk), $T_{\text {orb }}=2 \pi \sqrt{\left(G M_{s} / R^{3}\right)}=28$ years. Disks that undergo fragmentation are generally evolved for the shortest timescale (12 orbital times) because the growing local overdensities require increasingly smaller timesteps to be accurately followed, with the result of slowing down considerably the computation. We use up to 400, 000 timesteps for the most expensive calculations. Two simulations (DISL1 and DISgr) were carried out for a much longer timescale, about 20 and 30 orbital times, respectively, and their results will be described in section 3.5, together with the structural and orbital properties of the formed protoplanets.

All disks develop trailing spiral instabilities after a few rotations, the strength and nature of which depends on the $Q$ and $X_{m}$ parameters and on the equation of state. The disk expands because of the spiral arms, which shed angular momentum outwards and mass inwards; as a result its profile becomes more concentrated with time (Figure 4). Although initially the minimum $Q$ of the disks, $Q_{\min }$ is located at their outer boundary, their rapid expansion in response to the spiral instabilities causes a drop of the surface density in the outer part, and therefore $Q_{\text {min }}$ shifts further inward, near 13-14 AU (see Figure 1). The larger pressure gradients developing in adiabatic runs tend to erase disk substructure created by gravitational instability, while locally isothermal runs provide the most favourable conditions for the developing of the instabilities through damping of those same pressure gradients. The nature of the spiral pattern, namely which modes are dominant, depends on the details of disk structure. In general we observe that, for a given $Q$, disks with lower masses and temperature tend to produce higher order spiral patterns. This is likely related to swing amplification being stronger for higher order modes in lighter disks $\left(X_{m} \sim(\Sigma m)^{-1}\right)$ and has been previously observed by other authors (e.g. Nelson et al. 1998, 2000;Rice et al. 2003).

\subsection{Locally isothermal runs}

Disks evolved isothermally undergo fragmentation for $Q_{\min } \leq 1.4$. In these disks $Q$ drops below unity between 12 and 15 AU after $\sim 200$ years, and shortly after several clumps appear that become gravitationally bound $(2 P+U<0$, where $P$ is the pressure and $U$ is the gravitational binding energy) over a fraction of the orbital period, reaching densities of order $10^{7} \mathrm{~g} / \mathrm{cm}^{3}$ in their centers, up to 6 orders of magnitudes higher than the initial local 
density, their further collapse being limited only by numerical resolution (see section 3.3). When the local density grows beyond ten times the initial local value the gas should behave nearly adiabatically according to the radiative transfer calculations of Boss (2002) due to the increase in opacity (we recall that our disks have initial surface densities roughly identical to those in Boss' models) and therefore the locally isothermal approximation is no longer valid. Clump formation still proceeds when we switch to adiabatic conditions once the critical density threshold is reached. Clumps are fewer in the latter case but several gravitationally bound ones are still present (Figure 5). We note that our adiabatic conditions include irreversible heating from artificial viscosity — the temperature near the spiral overdensities indeed rises above the isothermal value, reaching $80 \mathrm{~K}$ - yet this is not enough to suppress clump formation at this stage because of the high density contrast already achieved (see Mayer et al. 2002). For $Q_{\min } \sim 1.65$ strong spiral arms are observed, but these saturate at some point (Laughlin, Korchagin \& Adams, 1997) reaching a near stationary pattern after almost 20 orbital times. At even larger values $Q_{\text {min }}, \sim 1.9$, very weak spiral arms form and then saturate. The evolution of the $Q$ parameter strongly suggests that the threshold between fragmentation and self-regulation must lie near $Q \sim 1.4$. Indeed models whose initial $Q_{\min }$ is 1.65 reach $Q_{\min } \sim 1.15$ locally (between 12 and $15 \mathrm{AU}$ ), which is only slightly higher than the $Q \sim 1$ required for fragmentation. A simulation with $Q_{\text {min }} \sim 1.5$ also did not lead to fragmentation (see Figure 6); the spiral arms reach a consistent amplitude after about 200 years, but then weaken and saturate. Therefore $Q_{\text {min }} \sim 1.4$ really seems to mark the threshold for fragmentation in our calculations, but in general such threshold will vary depending on the structural properties of the disk (e.g. its surface density profile).

As mentioned in section 2, the $Q$ profile is not enough to characterize the evolution of the different disk models; in addition to having different types of spiral patterns, disks with same $Q_{\text {min }}$ but different temperatures/masses yield clumps with varying mass. In particular, for a given $Q_{\text {min }}$ lighter and colder disks produce less massive clumps, but the mass of the clumps does not scale linearly with the mass of the disks. For example, model DISH3, which has a mass of $0.085 M_{\odot}$, produces several protoplanets with masses below a Saturn mass, which eventually grow up to a Jupiter mass or slightly above that (see below); therefore in this case protoplanets have masses up to 3-4 times smaller than those arising in disks only $15 \%$ more massive (e.g. model DISL1).

The trend can be understood in terms of the dependence of the local Jeans mass on disk temperature and mass. Only overdensities whose scale is above the Jeans length and below the Toomre critical wavelength (see Binney \& Tremaine 1987) will be able to grow and survive. In particular, any overdensity whose size is larger than the Toomre wavelength will be sheared away by differential rotation irrespective of disk temperature, and it will not grow in the first place unless its mass and size are above the local Jeans mass or length, 
respectively. Initially the Jeans length is comparable to the size of the entire disk, but as the instability proceeds and the disk midplane density increases it falls down to values below or around a Jupiter mass depending on the disk model. For disks having the same $Q_{\text {min }}$, the Jeans mass is smaller for lighter (colder) disks. In fact, from the definition of the Jeans mass we have $M_{J}=\frac{\pi}{6} \rho\left(\frac{\pi v_{s}^{2}}{G \rho}\right)^{3 / 2}$ ( $\rho$ is the density, $v_{s}$ the sound speed and $G$ the gravitational constant), hence $M_{J} \propto T^{3 / 2}$. But the scaling between density and temperature at fixed $Q$ is $\rho \sim T^{1 / 2}$ so ultimately $M_{J} \sim T^{5 / 4}$. A difference of a factor of 2 in the temperature (required to compensate a difference of order $\sqrt{2}$ in the disk mass) introduces a difference of a factor $\sim 2.4$ in the Jeans mass and hence in the minimum mass expected for condensations. Figure 7 shows that, indeed, there is a factor of 2 or more variation in the minimum mass of the clumps at the onset of fragmentation when we compare disks with different masses and same initial $Q_{\text {min }}$.

Is also the maximum size of the overdensities consistent with simple theoretical expectations? According to the tight-winding (WKB) approximation, which is only valid for tightly wound (local) perturbations in a differentially rotating thin disk, only density perturbations whose scale is smaller than the Toomre wavelength, $\lambda_{\text {crit }}=4 \pi^{2} G \Sigma / \kappa^{2}$, can grow. The most unstable wavelength is $\lambda_{m u}=0.55 \lambda_{\text {crit }}$ for zero-thickness gaseous disks (Binney \& Tremaine 1987). For most of our disks, for which $M_{d}=0.1 M_{s}$, we have $\lambda_{m u} \sim 5 \mathrm{AU}$ at distances between 12-16 AU, the region where fragmentation occurs, and because $\lambda_{m u} / R \sim 0.25$, i.e. $\lambda_{m u} / R<<2 \pi$ in the same range of radii, WKB results should still be valid for axisymmetric waves (Binney \& Tremaine 1987). For non-axisymmetric waves, the condition $X_{m}>>1$ (where $X_{m}$ is the parameter defined in section 2) is more appropriate, but this is also satisfied throughout most of the disk in the standard models, up to $m=3$ (but see below on the swing amplification). We would expect clumps to occur at scales of order $\lambda_{m u}$ and always below $\lambda_{\text {crit }}$. In disks with non-negligible vertical pressure, and hence finite thickness, both wavelengths will be somewhat smaller as the disk will have an effective self-gravity lower than in the truly thin case. Numerical softening, in addition, can also be thought as providing an artificial pressure on small scales. Romeo $(1992 ; 1994)$ has computed correction factors for the stability properties of self-gravitating stellar and gaseous disks which account for finite thickness and numerical softening. These factors are formulated as reduction factors for the surface density of the disks. In particular, if the wavenumber corresponding to the most unstable wavelength is $k_{m u}=2 \pi / \lambda$, and the typical disk scale height resulting from pressure is $h_{d}$, then the correction factor for finite disk thickness reads $\left(1+k_{m u} h_{d}\right)^{-1}$; an identical expression can be obtained for the softening $\varepsilon$, where $\varepsilon$ replaces $h_{d}$. In most of our simulations $\varepsilon<<h_{d}$, and hence we only consider the first reduction factor. Indeed, we find that $\lambda_{m u}$ is reduced by more than $60 \%$ once we include the latter factor, becoming $\sim 2$ $\mathrm{AU}$, which turns out to be the typical size of the banana-shaped overdensities that appear 
along the spiral arms just before fragmentation. These overdensities rapidly fragment into multiple clumps, but during this phase the system has become so strongly non-linear that any extrapolation of linear theory is meaningless. However, it is remarkable that while the system is still mildly non-linear the results of WKB theory are in good agreement with the simulations.

One ingredient that is not captured by the WKB approximation is swing amplification of non-axisymmetric modes. Indeed when strong swing amplification is expected it also means that linear theory breaks down (Binney \& Tremaine 1987). As we mentioned in the previous section, most of our disk models (e.g. DISL1, DISH1, DISH2) are barely in the regime where swing amplification is expected to be important at $R>10 \mathrm{AU}$. In particular, only for $m=3$ (or even higher order) modes the condition $X<3$, necessary for strong swing amplification (see Binney \& Tremaine 1987), becomes satisfied at some point and only quite late in the evolution (see Figure 3). However, model LIS4, that has a lower mass of the central star, clearly departs from this picture as $X<3$ for both $m=2$ and $m=3$ modes already early during evolution (this suggests that WKB results are less applicable here). Indeed the latter model exhibits a mixture of 3 and 2 armed modes visibly stronger than in the standard models ( 3 armed spirals are evident but two of the three arms grow more in amplitude and they begin to fragment, similarly to other simulations), as shown by comparing Figure 8

with Figure 6. Clumps appear almost 2 orbital times earlier in this model (the orbital time at $10 \mathrm{AU}$ is 40 years), after only 160 years, and the stronger non-axisymmetry produces orbits with eccentricities as large as $e=0.35$, thereby bigger than in the other models (see Mayer et al. 2002 and section 3.5 of this paper), which then favour more frequent and violent interactions between the clumps.

\subsubsection{The problem of the initial conditions}

How does fragmentation depend on the initial conditions of the disk simulations? An often heard argument against fragmentation models states that simulations with disks having a low $Q$ parameter by construction are unrealistic because the disk would respond to a developing gravitational instability by rising its temperature and adjusting its density profile, thus self-regulating to fairly high $Q$ values (Laughlin \& Bodenheimer 1994; Laughlin \& Roczyska 1996; Laughlin, Korchagin \& Adams 1997). The argument was originally developed against models starting with $Q$ near unity somewhere in the disk but one can easily imagine that mass redistribution due to milder non-axisymmetric instabilities might always drive the disk to values higher than even the critical threshold that we claim here, $Q \sim 1.4$. Moreover, in the simulations presented so far the disk has a sharp edge at $t=0$; although the disk very 
quickly (in one orbital time) expands as a result of its own evolution, initial reflection of waves at the edge might artificially activate a feedback loop and sustain swing amplification, leading artificially to a faster and stronger growth of the instability. Motivated by these arguments we performed a simulation (run DISgr in Table 1) in which the mass of the disk is increased gradually (over about 600 years, 20 orbital times at $R=10 \mathrm{AU}$ ) by a factor of 10 , going from $M_{d}=0.0085$ (well below even the minimum mass solar nebula) to $M_{d}=0.085$ (the final mass is the same as in model DISH3). The mass is increased uniformly in the disk at every step (in practice we grow the mass of each particle by a constant fixed). The disk is evolved isothermally in the first phase, and then adiabatically once the usual overdensity threshold is reached; the initial temperature profile is of the usual form (see section 2), and in particular the outer minimum temperature is $30 \mathrm{~K}$.

The disk stays considerably smooth for a long time (the initial $Q_{\min }$ is higher than 10), but as $Q$ approaches 2 spiral instabilities start to appear and grow in amplitude while the mass continues to increase (disk snapshots are shown in Figure 14), until $Q$ drops near unity (Figure 9) and clump formation occurs. Note that the disk surface density has not been redistributed significantly since fragmentation occurs at roughly the same temperature and the same mass as those initially assigned to one of the fragmenting standard models initialized with a low $Q_{\min }$, model DISH3 (see Table 1), This experiment shows that mass redistribution when $Q$ is higher than 1.4 or so is not important - non-axisymmetric torques are still too weak in this regime and the disk can still undergo clump formation provided that it remains cold. In addition, in this test the sharp outer edge disappears several orbital times before the first weak non-axisymmetric pattern becomes apparent in the disk, yet fragmentation takes place more or less as in the other runs. We only note that in this simulation the dominant spiral patterns are of higher order ( $m=5$ or higher) than in any of the other runs; this is in part due to the fact that the disk enters the regime of non-axisymmetric instability with a mass lower than in any of the other models, so that swing amplification is effective for high order modes only during the first part of the evolution (see Figure 9), but we cannot exclude that which is the dominant spiral pattern also depends on whether or not initial edge effects are present. The outcome of this run is in agreement with another result that points to negligible mass redistribution when $Q>1.5$; the fact that disks with $Q_{\min } \sim 1.65$ maintain a value of $Q_{\min }$ similar to the initial one after the strongest phase of the instability is over (see Figure 1). Of course in reality the process of mass accretion from the molecular cloud is much more complicated than depicted here; the disk mass will not increase monotonically but will probably reach a maximum and eventually decrease as accretion onto the central star or photoevaporation take over accretion from the molecular cloud (Matsuyama et al. 2003). In addition, and most importantly, the temperature will not remain constant but will change as a result of heating and cooling. 


\subsection{Adiabatic runs}

Disks evolved adiabatically since $t=0$ reach fragmentation only when starting from very low values of $Q_{\text {min }}$, as small as 0.8 . Such low values of $Q_{\min }$ are obtained with a combination of low temperatures and high masses $\left(M_{\text {disk }}=0.125 M_{\odot}\right)$ (see models marked "ad" in Table 1). With these very low values of the Toomre parameter the disk is locally unstable to axisymmetric perturbations. Indeed we see a ring forming in the outer part of the disks, but this is soon dissipated as $Q$ rises due to heating by compressions and shocks, and spiral arms form after 2-3 of orbital times. The precise value of $Q_{\text {min }}$ needed for fragmentation decreases with increasing values of $\gamma$ in the equation of state. The results are listed in Table 1 and shown in Figures 10 and 11. We note that even disks with $Q_{\min } \sim 0.8$ undergo fragmentation only if $\gamma=1.2$, which would happen only if some cooling is present. Also for $\gamma=1.3$ does fragmentation occur for $Q_{\text {min }} \sim 0.8$, but the clumps are quickly washed out by the strong developing pressure gradients, while for $\gamma=1.4$ overdensities in the spiral arms are washed out before being able to collapse. The temperature rises to more than $100 \mathrm{~K}$ along the spiral arms due to compressional and shock heating. Therefore, even at these very low values of $Q$ we do find that fragmentation depends on the equation of state, while an early work by Boss (1998) was finding fragmentation even for $\gamma=1.4$ with $Q_{\min } \sim 1$. Our tighter limits are likely due to the inclusion of irreversible shock heating, which was absent in that as well as in other studies, and is instead important in these violently unstable models. Shock heating is evident along the trailing and leading edges of the spiral arms (see the temperature maps in Figure 11); indeed these are the locations where the velocities of the gas in the spiral arms and the mean sound speed differ the most.

As we mentioned above, these very low $Q$ states are probably unrealistic,and this is shown by the rapid evolution that the disk undergoes, with $Q_{\min }$ rising by a factor of 2 in less than 5 orbital times. The clumps that form in run DISad4 are particularly big (see section 3 on how disk mass and temperature influence clump formation) growing to more than 10 Jupiter masses after a few mergings. Finally, we note that in adiabatic simulations that start from higher values of $Q_{\min }$ (DISLad1,DISLad5,DISLad6 in Table 1), fragmentation does not take place. The temperature along the spiral arms, on the other end, only grows from 56 to about $70-80 \mathrm{~K}$ because the non-axisymmetric modes are much weaker than in the runs with lower $Q_{\text {min }}$; this temperature is only $\sim 50 \%$ higher than that required to maintain $Q \sim 1.4$ and follow the path towards strong instability (the change in temperature has a negligible dependence on the magnitude of the artificial viscosity, see Table 1 for the different cases considered). Therefore shock heating is not dramatic and only little cooling would be needed to bring the disk towards the marginally unstable regime; on the rise in temperature associated with shock heating occurs very fast, over less than an orbital time, so it remains to be seem whether cooling processes can act at comparable speed (but see 
Boss 2002a).

\subsection{Mass and force resolution; how they affect clump formation and evolution}

The physical interpretation of fragmentation proposed in section 3.1 clearly implies that the simulations ought to resolve the Jeans and Toomre wavelengths. The ability to resolve these characteristic physical scale lengths depends on both mass and force resolution in an SPH simulation; in particular, resolution must be high enough to resolve the smallest among the two scales, usually the Jeans length. As extensively discussed by Bate \& Burkert (1997), the gravitational softening and the SPH smoothing length should be comparable and both smaller than the local Jeans length for the calculations to be trustworthy (see also Nelson 2003). When the gravitational softening exceeds the SPH smoothing length any eventual collapse will be slowed down or halted, while artificial fragmentation might occur in the opposite situation. Bate \& Burkert also showed that the local Jeans mass should be always resolved by no less than $2 N_{\text {neigh }}$, where $N_{\text {neigh }}$ is the number of neighbors used in the SPH smoothing kernel, $N_{\text {neigh }}=32$ in our runs. Thanks to the extremely high resolution adopted in this work, the local Jeans mass is always resolved by several hundred to several thousand particles, hence we are orders of magnitude above the minimum requirements. On the other end, shortly after clump formation the smoothing length becomes significantly shorter than the gravitational softening inside the clumps and hence the collapse of the clumps is ultimately slowed down once they have shrunk down to a scale comparable to the softening, which then acts like an artificial pressure force. This means that in the isothermal calculations the clumps become effectively super-adiabatic near the center, where the resolution limit is reached - the difference with the calculations in which the equation of state is switched to adiabatic exists only in the very first stage of clump formation, when the softening is still comparable to the other relevant scale lengths. The bottom line is that any detailed analysis of the internal structure of the clumps must be postponed to future simulations with even higher resolution. For the moment only mean properties of the clumps, calculated as averages over the entire systems (which is comfortably larger than the softening) can and will be discussed (see section 3.5).

A very important result emerging from our set of simulations is that clump formation is enhanced with increasing mass resolution for the same initial conditions (with the softening

scaling as $m_{g}{ }^{1 / 3}$; a larger number of clumps is seen at higher resolution, although the mass scale of fragmentation is basically unaffected (Figure 12). Indeed the minimum clump mass depends on the local Jeans mass, which in turn is determined by the disk density and temperature, not by resolution (section 3.1). Force resolution alone also has an effect. A 
softening larger or comparable to the maximum unstable Toomre wavelength suppresses clump formation even in unstable disks; in fact a large softening acts as an additional pressure (in practice it suppresses gravity) at the crucial scale where the perturbation has the highest amplitude, or, alternatively, one can view it as producing an effective increase in the local Toomre parameter. Following Romeo (1994), the minimum allowable softening for our disks should be around $0.37 \mathrm{AU}$, which, of course, is close to the effective $\lambda_{m u}$ calculated in section 3.1. Actually we find that a softening $\sim 3$ times smaller than the latter is necessary to go beyond the stage of the mildly non-linear regime and enter that of clump formation (see Table 1). Indeed the calculation done by Romeo applies to the study of spiral structure in marginally unstable (galactic) disks and not to the strongly unstable regimes that we are investigating here; when $Q$ locally drops below 1 and the strongly non linear regime is reached a higher force resolution, close to the rapidly dropping Jeans length, should be required in order to keep following the dynamical evolution properly. Therefore, in a simulation both mass and force resolution must be high enough to follow the fragmentation process in the disk. The maximum and minimum allowable softenings can be accurately determined only through convergence tests, the only a priori prescription being to balance softening and smoothing length (Bate \& Burkert 1997). We note, however, that the line dividing stable and unstable disks is only weakly dependent on softening in our simulations; disks that are stable with our "standard" choice of parameters remain stable even with a softening ten times smaller (for example compare run DISH4 and DISH4b in Table 1). This means that the threshold for stability, $Q_{\min } \sim 1.4$, is a robust physical result, at least under the thermodynamical conditions adopted.

\subsection{Dependence on artificial viscosity}

We find that both the value of $\alpha$ and $\beta$ have some impact on disk evolution and fragmentation. Whereas disks that do not fragment are found to behave so irrespective of the artificial viscosity, disks that fragment do so more or less severely and on slightly different timescales depending on the value of these parameters. Here we discuss the results of locally isothermal runs with varied viscosity parameters (all listed in Table 1), hence we neglect the artificial heating eventually induced by viscosity. The latter is present in adiabatic runs, but we already showed that in such runs disks never form bound clumps unless we reduce the value of $\gamma$ significantly below the canonical 1.4 (see section 3.2), which makes them rather unsuitable for analyzing the effects of viscous heating on clump formation. Therefore we decide to postpone the analysis of artificial viscous heating to a forthcoming paper in which we implement also radiative heating and cooling in the disks (Mayer et al., in preparation). 
In general a smaller value of $\beta$ or $\alpha$ enhances fragmentation and the opposite happens for larger values. Only $\beta \geq 3$ can completely suppress fragmentation; however such high values of $\beta$ are not a good choice for flows with moderate Mach numbers $(\sim 1-1.5)$ like those occurring in our simulations (see e.g. Hernquist \& Katz 1989; Thacker et al. 2000) but could mimic the behaviour of disks with a high degree of turbulence (Nelson et al.1998). Why is viscosity affecting clumping? In general artificial viscosity makes the velocity and density fields smoother, which helps to reduce post-shock oscillations and noise but could in principle suppress small-scale physical features in the velocity field of the fluid. When the collapse of an overdensity begins, particles locally acquire radial motions, and hence the radial velocity dispersion will rise; a high viscosity can damp these radial motions and hence the collapse. The fact that the disk velocity dispersion profile is both lower and smoother with higher artificial viscosity is an indication of such an effect (Figure 13).In particular, disk models that produce several bound clumps with the standard values of artificial viscosity (DISL1), clearly have their localized peaks in the radial dispersion profile completely smeared out for higher viscosity (DISL1e and DISL1f). The question arises whether the dependence on artificial viscosity is strong enough to change the threshold $Q$ for fragmentation. The answer is negative based on our simulations - disks with $Q$ above the threshold remain stable irrespective of the values of $\alpha$ and $\beta$. This is highlighted by the comparison between runs DISH2 and DISH2b , in which a disk with initial $Q_{\min }=1.65$ is evolved first with the standard parameters and then with $\alpha=0$ and $\beta=0.5$; while the velocity dispersion profiles look somewhat different, the dynamical evolution is substantially identical, and in both runs the disk does not fragment.

\subsection{Long term evolution of disks and protoplanets}

Once the disks enter the fragmentation phase timesteps become extremely small locally, and the computation becomes extremely demanding. One million particle runs require almost 400,000 steps up to 350 years of evolution, $80 \%$ of which cover only the last 2-3 orbital times ( 100 years), after clumps begin to form. We resort to lower resolution runs $(200,000$ particles) to probe the disk evolution over a more extended time (see also Mayer et al. 2002). In these runs the thermodynamics switch to adiabatic as soon as the local density becomes ten times higher than the initial value (see section 3.1). The central temperature of the clumps grows up to $300-400 \mathrm{~K}$ rapidly after formation takes place owing to strong compression while their collapse proceeds on their internal dynamical time (of order of days) ; however, the clumps would certainly become much hotter in the center if their collapse was not halted at a scale comparable to the gravitational softening. Two low-res simulations ( $N=200,000$ particles), one for a disk with mass $M=0.1 M_{\odot}$ (same as in run DISL1) and one for the 
growing disk (run DISgr, final mass $M=0.085 M_{\odot}$ ) were run for, respectively, 30 and 20 more orbital times (the reference orbital time being measured at $10 \mathrm{AU}$ ) after the onset of the fragmentation. Due to their extremely high densities protoplanets are never destroyed by the tidal field of the central star (their tidal radius is more than ten times larger than their typical size) but are tidally perturbed by and undergo a series of mergers with neighboring protoplanets (this phase lasts about 10 orbital times) until only 3 and 2 protoplanets remain in, respectively, model DISL1 (see also Mayer et al. 2002) and model DISgr (see last snapshot of Figure 14 for run DISgr). In both simulations protoplanets have eccentric orbits, with the eccentricity, $e$, running between 0.1 and $0.3\left(e=\left(R_{\text {apo }}-R_{\text {peri }}\right) /\left(R_{\text {apo }}+R_{\text {peri }}\right)\right.$, where $R_{\text {apo }}$ and $R_{\text {peri }}$ are, respectively, apocenter and pericenter distance.) and end up at mean distances between 3 and $12 \mathrm{AU}$. These eccentricities correspond to the mean values found for extrasolar planets (Marcy \& Butler 1998; Marcy et al. 2000). Larger eccentricities (comparable to the highest measured for extrasolar planets, $\sim 0.7$ ) are measured in a run in which the same disk as in run DISL1 is evolved with a locally isothermal equation of state throughout the evolution (see below) and, in general, could result from dynamical relaxation of these systems of massive protoplanets in several hundred thousand years (Terquem \& Papaloizou 2002; Papaloizou \& Terquem 2001). The gravitationally bound masses of the planets remaining at the end of the simulations (calculated by considering all particles for which $2 P+U<0$, where $P$ is the pressure and $U$ is the gravitational binding energy) range between 2.4 and $6.6 M_{J}$ in the higher disk mass case, and between 0.07 and $1.7 M_{J}$ in the lower disk mass case. Hence both super-Jupiters and planets with masses as small as that of Saturn seem to be a possible outcome of the instability mechanism. This is an important feature of the model - its natural flexibility in accounting for the entire range of masses of gas giants known so far.

The final masses of the planets are not just the result of merging but also of accretion of ambient gas, and the accretion rate in turn depends on the ambient pressure and thus on the equation of state of the gas (D'Angelo, Kley \& Hennings 2003). In the extended simulations the equation of state is normally adiabatic, which means high pressure support of the surrounding gas (no cooling), and thus should yield a lower limit on the accretion rate. The mean accretion rate measured over the 500 years following the onset of fragmentation in the run employing model DISL1 is quite low, in the range $10^{-7}-10^{-6} M_{\odot} / y r$ (different accretion rates are found for the different planets, in particular the lowest are found for the planet closest to the star, as expected from the higher ambient pressure). The accretion rate is declining towards the end of the simulations, and during the last couple of orbital times is practically zero. Therefore, at least in these adiabatic runs the measured protoplanetary masses after $\sim 1000$ years are certainly a good estimate of the final ones. The mass accretion rate onto the central star, instead, is still high even at the end of the simulations, being 
$>10^{-6} M_{\odot} / y r$ (see Figure 16); using the value of the accretion rate in the last stage of the simulation would yield a disk dissipation timescale as small as 20000 years (this is likely a lower limit given that the accretion rate is still declining at the end).

In passing we note that the short disk dispersal timescales predicted here as a result of gravitational instability would solve the puzzle of short disk lifetimes (shorter than a million year in at least 30\% of stars in Taurus, see Armitage, Clarke \& Palla 2003). Other solutions, like photoevaporative flows (Clarke, Gendrin \& Sotomayor 2001; Armitage, Clarke \& Palla 2003) require an input from external irradiation sources since the heat generated by internal star-disk accretion shocks is probably insufficient (Matsuyama, Johnstone \& Hartmann 2002), but this, for example, would not work in Taurus because there are no massive stars capable of generating such a strong photoevaporating flux. One might worry that the accretion rate of gas towards the center, and, in general, any motion of the particles in the disk, might be partly caused by the artificial viscosity, which is well known to produce spurious losses of angular momentum (Thacker et al. 2000). We tested this latter possibility by stopping mass accretion in model DISgr when $M_{d}=0.01 M_{\odot}$ (the mass is an order of magnitude smaller than our typical disk masses) and running it forward in time for 20 orbital times. The disk remains very smooth in this case $\left(Q_{\min }>8\right)$, and the accretion rate towards the end is nearly two orders of magnitude lower than in the other runs, being stationary at around $3 \times 10^{-7} M_{\odot} / y r$. We interpret the last number as the residual accretion rate due to artificial viscosity (note that this is a conservative choice because the disk is never perfectly axisymmetric due to the inevitable Poisson noise in the initial conditions); the corresponding accretion timescale (defined as the time required by a massive disk of about $0.1 M_{\odot}$ to accrete onto the star by the latter mechanism only) is close to half a million year, so much longer than any of the timescales considered here. Hence the fact that a high accretion rate is a necessary consequence of gravitational instability and provides a way to clear out the disk very rapidly seems a well grounded inference. Indeed, Pickett et al. (2003) have recently obtained similar accretion rates (although they probed the disk evolution on a shorter timescale) using high resolution grid-based simulations that have a much lower numerical viscosity. Notwithstanding the high mass of the protoplanets, most of the mass at the end of the simulations, about $70 \%$, is still in the disks; of this mass almost $30 \%$ is accumulated in the inner 2-3 AU as a result of accretion triggered by the non-axisymmetric torques and, partly, by artificial viscosity, while the rest is still at $R>5 \mathrm{AU}$, and hence will be still important in determining the orbital evolution (and eventual migration) of the planets (see Lufkin et al. 2003). Accretion rates peak at values higher than $10^{-5} M_{\odot} / y r$, significantly in excess of the rate measured in T Tauri stars (Gullbring et al. 1998); the strong bursts of infrared luminosity found in disks caught during the early stages of their evolution, like FU Orionis, are suggestive of such high accretion rates and might indeed be 
explained with such strong inflows due to gravitational instability (Lodato \& Bertin 2003).

We tested how accretion rates depend on the equation of state by re-simulating for a few orbital times model DISL1 with a locally isothermal equation of state during and past fragmentation. The protoplanetary masses are four times higher than in the adiabatic simulation after 600 years (at this point we stop the simulation), the time-averaged accretion rate being about 50 times higher. By this time, however, planets have also carved gaps that are not present in the adiabatic runs (compare Figure 15 with last snapshot of Figure 14); although material can still flow to the planet, the accretion rate is strongly reduced after gap formation (Bryden et al. 1999), in particular is about a hundred times smaller than the rate at which gas accretes onto the central object; therefore the disk will be dissipated well before the planets can grow significantly further (Figure 16). Once planets have carved a gap, further accretion of disk material should occur on the viscous timescale; the only physical source of viscosity in our disks is self-gravity (Lin \& Pringle 1987; Laughlin, Korchagin \& Adams 1997) because, by design, we lack other possible sources like, for example,magnetic fields. The effect of artificial viscosity (Bryden et al. 1999), on the other end, is negligible at our resolution, as we discussed above. Therefore, at this point the accretion rate onto the protoplanets should in principle be proportional to that onto the central star. However, not everywhere in the disk is the dominant bulk motion directed towards the center. In fact, the direction of the torques arising from gravitational instability changes with radius, and, typically, while the material in the inner regions loses angular momentum, the material in the outer part gains it. The transition radius is typically set by where the dominant unstable modes (the spiral arms in our case) occur, which in our simulations is always between 10 and $15 \mathrm{AU}$ from the center; therefore we expect that the protoplanets located at $R<10 \mathrm{AU}$ should accrete much more mass and much more rapidly than those located at $R>10 \mathrm{AU}$ (the actual accretion rate will be determined by the local flux of mass around the protoplanet, hence by the local details of the gravitational torques). The final masses of the protoplanets in this last run are between 10 and $25 M_{J}$, therefore intermediate between those of brown dwarfs and those of extrasolar planets (Udry et al. 2002). However, we believe that these numbers are not to be taken seriously. Indeed, by marking particles belonging to a clump at the final time and tracing them back we found that most of the material accreted by the protoplanets comes from the midplane and usually from a narrow annulus coplanar with the orbit of the planet in the adiabatic runs, while it occurs in a much more isotropic fashion in the isothermal runs, with a large fraction of accreted particles originally located at high distances from the plane; this big difference is certainly due to the fact that the vertical pressure gradients and those across the spiral shocks near the planet are artificially low in the isothermal simulation (shock heating is instantaneously damped). In fact, after 600 years of evolution the vertical structure of the isothermal and adiabatic run are dramatically 
different, the scale height being almost ten times as big in the latter. This is reflected in the comparison between the vertical temperature profiles (Figure 17).

In general, a conclusive answer on the final masses of the planets formed via gravitational instability has to await a more realistic treatment of the disk thermodynamics, with heating and cooling correctly model both inside and outside the overdense regions (Mayer et al., in preparation).

There are hints that protoplanets undergo some orbital migration (Mayer et al. 2002), but this does not seem to have a preferred direction due to the chaotic nature of the torques present in the system (as a result of both the non-axisymmetric global potential and the gravitational interactions with the other planets). This supports the idea that current models of planet migration become unrealistic once non-trivial disk profiles (Artimowicz \& Peplinski, in preparation) and interactions between several bodies are simultaneously taken into account (Lufkin et al. 2003). Although the protoplanets are massive, the ratio of their masses to that of the disk left after nearly a thousand years of evolution is still as small as a few times $10^{-2}$ in the adiabatic run; therefore (Ward 1997a,b; Tanaka et al. 2002) we do not expect migration to occur on the viscous timescale of the disk as in the classic Type II regime (gap formation is not apparent indeed) instead gravitational torques should be still the dominant source like in the Type I regime, albeit with the additional complication of disk self gravity and mutual interaction between the protoplanets. On the contrary, in the isothermal run the protoplanets acquire much higher masses due to the higher accretion rates, gaps are carved by them and the orbital evolution resembles that of the Type II regime (see above) with the difference that the disk is being dissipated very quickly. Whereas we are not able to probe the systems for timescales long enough to draw conclusions, an efficient inward orbital migration is hard to imagine in the traditional framework because the disks acquire a significantly steeper inner density gradient (inside the orbits of the planets) due to accretion of gas onto the central star — outward migration should thus be more likely (Masset \& Papaloizou 2003).

So does this mean that hot Jupiters are difficult to explain within the present model? Not necessarily. There are indeed several possibilities to have efficient migration as soon as we move out of the standard framework. Indeed, one of the mechanisms recently proposed to explain the observed orbital distribution of extrasolar planets relies on dynamical relaxation of a population of massive planets formed rapidly through gravitational instabilities (Papaloizou \& Terquem 2001). This latter model assumes that the disk is dissipated on a timescale much shorter than that required for relaxation (the latter being of order of thousand of orbits, or several tens of thousand years for the orbital timescales typical of our simulations), an assumption that seems to be marginally supported by our simulations. 
As mentioned above, when the disk is still present, the net gravitational torque caused by the instability will have different directions depending on the location in the disk. In the locally isothermal run with model DISL1 there is one protoplanet significantly inside 10 AU, and this might sink towards the center in only a few thousand years if it just follows the "bulk" accretion flow estimated for the disk (Figure 16). The same would happen even in the adiabatic run, but of course the sinking timescale will be longer this time, of order of a few tens of thousand years. Therefore, one could speculate that if protoplanets can migrate inwards just enough to find themselves inside the region where the torques due to gravitational instability become negative then they could drift towards the center rapidly together with the rest of the disk, eventually stopping at some distance from the star only after the non-axisymmetric torques have faded away; of course how much the planet can sink thanks to this latter mechanism is completely undetermined at the moment, but future simulations are on the way that will probe much longer timescales and possibly provide the answer.

Aside from the latter possibilities, certainly a more straightforward prediction of our model is that inward migration should not be very efficient; this is actually a good feature of disk instability — that of planets sinking too fast towards the central star has become an increasingly hard problem to solve in the past few years within the framework of the standard core-accretion model (e.g. Bate et al. 2003). Ways that have been proposed to halt migration, like the interaction between a planet on an eccentric orbit and the surrounding disk (Papaloizou \& Larwood 2000; Papaloizou 2002) are naturally included in the gravitational instability model studied here, although the individual effects are hard to disentangle given the complexity of the evolution. On the other hand, we have to keep in mind that our simulations still lack several ingredients like stellar winds, photoionization and magnetic turbulence (Balbus \& Hawley 1991; Nelson et al. 2003; Matsuyama, Johnstone \& Murray, 2003) that can affect substantially the inner disk, eventually creating cavities or severely affecting the density profile, with consequent effects on the speed and direction of migration.

The surviving protoplanets are differentially rotating, nearly spherical bodies slightly flattened by rotation (the ratio between major and minor axis is $\sim 0.9$ - see Figure 18). We measure equatorial rotation speeds (this is measured at the outermost radius for which particles are gravitationally bound to the clump) and then calculate a tentative final rotation speed of the protoplanets by allowing contraction down to the mean density of Jupiter assuming conservation of angular momentum. We find values in the range $3.5-40 \mathrm{~km} / \mathrm{s}$, which nicely encompass the (equatorial) rotation speeds measured for Saturn and Jupiter, 8.7 and $14.6 \mathrm{~km} / \mathrm{s}$ respectively. We note that a long standing problem has always been how Jupiter can still maintain a high rotation speed despite the fact that some kinetic energy must have been dissipated by atmospheric friction after its formation; the present model suggests a solution in that protoplanets could form with rotation speeds well in excess of 
the speed that Jupiter has today. Among the surviving protoplanets the obliquities go from a few degrees to 160 degrees. Large obliquities seem to be associated with mergers (and thus transfers of angular momentum); the planet experiencing fewer mergers is in both runs that with the smaller obliquity. Our model would then naturally explain the wide range of obliquities that we find in our Solar System once we consider gas and ice giants together (a possible common origin of gas and ice giant planets via gravitational instability is discussed in Boss 2002b and Boss, Wetherill \& Haghighipour 2002).

\section{Conclusions}

The main result shown in this paper is that fragmentation into long-lived, tidally stable, gravitationally bound protoplanets with masses and orbits comparable with those of observed extrasolar planets is possible in marginally unstable protoplanetary disks $\left(Q_{\min }<1.4\right)$. The requirement is that the disk can cool efficiently (as implicit in the locally isothermal approximation) until the spiral arms approach fragmentation; once the the local density grows by roughly an order of magnitude gravity is strong enough for the collapse to proceed even with purely adiabatic conditions. We also showed that resolution, both in mass and in the gravitational force calculation, is a decisive factor in order to model disk evolution and fragmentation properly. In SPH simulations, in particular, it is crucial that gravity and pressure be resolved at comparable levels for most of the extent of the simulations. In fact, no matter how many particles are used, if the gravitational softening is too large, the spiral arms do not reach the critical amplitude for fragmentation as the dynamical response of the system is altered - growing modes are suppressed. Once fragmentation is approached, a high resolution is also needed for the clumps to continue collapsing. Because the survival of clumps subject to strong tidal stresses depends on their binding energy, thus on the density they are able to reach, the fact that in our simulations clumps survive for several tens of orbital times is also a consequence of the high resolution employed. Their densities are several order of magnitudes higher than the mean density, resulting in a tidal radius ten times larger than their typical size. Therefore they will eventually survive for timescales much longer than those probed here and can thus be associated with protoplanetary objects. Their destruction can only result through mergers with other protoplanets or from a strongly increased tidal field in case their orbit migrates inward substantially. Pressure gradients near the clumps might drive dust and planetesimals and enrich the gaseous protoplanets up to metallicities beyond the solar value (Haghighipour \& Boss 2003).

Previous works on the gravitational instability fell short of the resolution needed to follow the very non-linear stage of disk evolution. In addition, fixed boundaries were certainly 
a problem; in run DISL1, for example, both the outermost and the innermost clump go, respectively, further out and further in than the initial outer and inner radius of the disk, due to their eccentric orbits; therefore, even with enough resolution typical fixed grids would have not been able to follow 2 out of 3 clumps (see also Pickett et al. 2000a and Boss 2000). Recently, Pickett et al. (2003) identified several "banana-shaped" overdensities in their grid simulations; these structures have densities and shapes strikingly similar to what we find in our disks just before clump formation starts. As they discuss and test, their simulations seem to lack enough azimuthal resolution to be conclusive about the evolution of the overdensities. As a comparison their grid cell size is $\sim 5$ times bigger than the gravitational softening in our $10^{6}$ particles runs in the outer, more unstable regions; indeed in runs where the gravitational softening is increased by a factor of 3 or more with respect to the nominal value (DISL1b,c in Table 1) we also witness a suppression of fragmentation (section 3.3).

Whereas the global non-axisymmetric instabilities seen in the simulations and the resulting fragmentation cannot be captured by the WKB approximation, the maximum scales of the overdensities in the mildly non-linear regime seem to be understandable in terms of the maximum Toomre wavelength. The minimum masses of the forming clumps are instead controlled by the local Jeans mass; because of the scaling with disk mass and temperature, disks with similar $Q$ profiles can produce smaller or bigger clumps depending on their mass and temperature. The smallest clumps have masses lower than that of Saturn at formation. Further mass growth due to merging and accretion shifts the typical masses to objects comparable or bigger than Jupiter, but still it is clear that gravitational instability does not produce only super-Jupiters. Indeed, the mean mass among the surviving protoplanets in the extended runs is $\sim 2.5 M_{j}$.

We investigated whether starting a disk with a low $Q_{\min }$ at the beginning of a simulation, as we always do, might artificially enhance fragmentation. We showed that a disk grown from a very light state over tens of orbital times still produces several gravitationally bound protoplanets once it reaches values of temperature and mass comparable to the initial ones of a model that undergoes clump formation (section 3.5). This latter result as well as the fact that disks with $Q_{\min }$ just above the threshold for fragmentation have a $Q$ profile similar to the initial one after the instability saturates (Figure 1), suggest that the non-axisymmetric instabilities occurring when $Q_{\text {min }}>1.4$ are too weak to redistribute as much mass as needed to suppress the instability; a little cooling will easily bring the disk towards a marginally unstable state with $Q_{\min }<1.4$. Therefore $Q_{\min } \sim 1.4$ appears to be a significant threshold. In reality the growth of the disk mass with time will be determined by the balance between the accretion rate onto the central star as determined by both gravitational instability and other processes, for example viscosity produced by magnetic fields, and the accretion rate of material falling onto the disk from the molecular cloud envelope. Therefore the mass will not 
grow at a constant rate as assumed here, instead the process will be strongly time dependent; however, hydrodynamical simulations of disk formation (Yorke \& Bodenheimer 1999), that include radiative transfer but neglect magnetic fields, do find that disks reach $Q$ parameters in the range $1.3-1.5$ early in their evolution. Whether fragmentation will actually occur will then depend on how well a disk can radiate away the thermal energy produced by compression and shocks along the edges of the growing spiral arms. This is the most important, still open question concerning the final outcome of gravitational instabilities with the inclusion of realistic thermodynamics (Pickett et al. 1998,2000a,b, 2003; Meija et al. 2003). In fact, even in our growing disk simulation we were keeping the local temperature constant before the appearance of the overdensities. Recent, lower resolution SPH simulations that solve for heating and cooling find that fragmentation can proceed when the cooling time is comparable to the disk orbital time (Rice et al. 2003), basically confirming previous simpler numerical and analytic calculations by Gammie (2001). Similar conclusions are reached in the recent three-dimensional calculations with volumetric cooling by Pickett et al. (2003). Such short cooling timescales are actually achieved in the simulations of Boss (2001, 2002a,b), that include radiative transfer in the diffusion approximation with realistic disk opacities, due to efficient vertical energy transport by convection. We will address these issues in a forthcoming paper using very high resolution simulations which incorporate different forms of radiative cooling. These simulations will also allow to model more realistically the accretion rate onto the protoplanets and thus produce a better prediction for their masses.

Finally, within the gravitational instability model the appearance of the protoplanets and a rapid disk dispersal seem to be linked; although it is premature to estimate a robust disk dispersal timescale (even this can vary depending on the way the disk thermodynamics is treated), our calculations suggest that most of the disk material originally at tens of AU from the central star will be accreted in less than $10^{5}$ years. Material originally located outside the strongly unstable region, say at distances of about $100 \mathrm{AU}$, would gain the angular momentum shed by the strong spiral arms and avoid rapid accretion; therefore a prediction of the gravitational instability model is that there must be a population of fairly young protoplanetary disks (considerably less than a million years old) in which a gap in the mass density of gas exists over a few tens of AU between an inner and an outer zone. SIRTF and other upcoming missions (Evans et al. 2003), by looking in the mid-infrared wavelengths, will allow for the first time to trace the structure and evolution of the gaseous component in the protoplanetary disks using the rotational emission lines of its main constituent, molecular hydrogen, and will provide a direct estimate of disk dispersal timescales.

L.M. thanks all the participants to the workshop "Circumstellar disks and protoplanets" organized by Tristan Guillot at the Nice Observatory in February 2003, in particular 
Hal Levinson, Doug Lin, Pawel Artimowicz, Tristan Guillot, Paolo Tanga, Patrick Michel, Alessandro Morbidelli and Ricardo Hueso for insightful and stimulating discussions. The numerical simulations were performed on LeMieux at the Pittsburgh Supercomputing Center,

on the Z-Box at the University of Zurich and on the Intel cluster at the Cineca Supercomputing Center in Bologna (Italy).

\section{REFERENCES}

Armitage, P.J., Clarke, C.J., \& Palla, F., 2003, MNRAS, 342, 1139

Armitage, P.J., \& Hansen, B.M.S., 1999, Nature, 6762, 633

Artymowicz, P., Lubow, S. H., and Kley, W., 1998, in "Planetary systems - the long view", Eds. Celnikier, L. et al., Editiones Frontieres, pp. 381-389

Balsara, D.S., 1995, J. Comput. Phys., 121, 357

Balbus, S., \& Hawley, J.F., 1991, ApJ, 376, 214

Barnes, J., \& Hut, P., 1986, Nature, 324, 446

Bate, M.R., \& Burkert, A., 1997, MNRAS, 288, 1060

Bate, M.R., Lubow, S.H., Ogilvie, G.I., \& Miller, K.A., 2003, MNRAS, in press

Beckwith, S.V.W., Sargent, A.I., Chini, R., \& Gusten, R., 1990, AJ, 99, 924

Binney, J., \& Tremaine, S., 1987, Galactic Dynamics (Princeton University Press: Princeton)

Boss, A.P., 1993, ApJ, 417, 351

Boss, A.P., 1996, ApJ, 469, 906

Boss, A.P. 1997, Science, 276, 1836

Boss, A.P., 1998, ApJ, 503, 923,

Boss, A.P., 2000, ApJ, 545, L61

Boss, A.P., 2001, ApJ, 563, 367

Boss, A.P., 2002a, ApJ, 576, 462

Boss, A.P., 2002b, E\&PSL, 202, 513 
Boss, A.P., Wetherill, G.W., \& Haghighipour, N., 2002, Icarus, 156, 291

Bryden, G., Chen, X., Lin, D.C.N., Nelson, R.P., \& Papaloizou, J.C.B., 1999, ApJ, 514, 344

Briceno, C. et al. 2001, Science, 291, 93

Cameron, A.G.W., 1978, Moon Planets, 18, 5

Charbonneau, D.T., Brown, T.M., Latham, D.W., \& Mayor, M., 2000, ApJ, 529, L45

Clarke, C.J., Gendrin, A., \& Sotomayor, M., 2001, MNRAS. 328, 485

D’Alessio, P., Calvet, N., \& Hartmann, L., 2001, ApJ, 553, 321

D’Angelo, G., Kley, W., \& Henning, T., 2003, ApJ, 586, 540

Dutrey, A., Guilloteau, S., Duvert, G., Prato, L., Simon, M., Schuster, K., \& Menard, F., 1996, A\&A, 309, 493

Evans, N.J.,II, et al., 2003, PASP, 115, 965

Gammie, C.F., 2001, ApJ, 553, 174

Guillot, T., 1999a, Planet. Space Sci., 47, 1183

Guillot, T., 1999b, Science, 286, 72

Guillot, T., \& Showman, A.P., 2002, A\&A, 385, 156

Gullbring,E., Hartmann, L.,Briceno, C., \& Calvet, N., 1998, ApJ, 492, 323

Haghighipour, N., \& Boss, A.P., 2003, ApJ, 586, 144

Haisch, K. R. jr., Lada, E. A., \& Lada, C.J., 2001, AJ, 121, 2065

Hernquist, L., \& Katz, N., 1989, ApJS, 70, 419

Hubickyj, O., Bodenheimer, P., \& Lissauer, J.J., 2002, American Geophysical Union, Fall Meeting 2002

Kawakita, H., et al. 2001, Science, 294, 1089

Kuiper, G., 1951, Proc. Natl. Acad. Sci., 37, 1

Laughlin, G. \& Rozyczka, M., 1996, ApJ 456, 279

Laughlin, G., \& Bodenheimer, P. 1994, ApJ, 436, 335 
Laughlin, G., Korchagin, V., \& Adams, G., 1997, ApJ, 477, 410

Lin, D.N.C., \& Pringle, J.E., 1987, MNRAS, 225, 607

Lin, D.N.C, \& Papaloizou, J.C.B., 1979, MNRAS, 188, 191

Lin, D.N.C., \& Papaloizou, J.C.B., 1993, in Protostars and Planets III, eds, E.H. Levy, \& J.I. Lunine (Tucson:University of Arizona Press), p. 749

Lissauer, J.J., 1993, ARA\&A, 31, 129

Lodato, G., \& Bertin, G., 2003, A\&A, 408, 1015

Lufkin, G., Quinn, T., Wadsley, J., Stadel J., \& Governato, F., 20 03, astro-ph/0305546

Masset, F.,\& Papaloizou, J.C.B., 2003, ApJ, 588, 494

Matsuyama, I., Johnstone, D., \& Murray, N., 2003, ApJ, 585, L143

Mayer, L., Quinn, T., Wadsley, J., \& Stadel, J.J., 2002, Science, 298, 1756

Mayor, M., Udry, S. \& Queloz, D., 1999, AAS/Division for Planetary Sciences Meeting, 31

Monaghan, J.J., \& Gingold, R.A., 1983, J. Comput. Phys., 52, 374

Marcy, G.W., \& Butler, R.P., 1998, ARAA, 36, 57

Marcy, G.W., Vogt, S., Fischer, D., \& Butler, R.P., 2000, AAS, 196, 3514

Meija, A.C., Durisen, R.H., \& Pickett, B.K., 2003, in ASP Conf. Ser. 294, Scientific Frontiers in Research on Extrasolar Planets, ed. D.Deming (San Francisco:ASP), in press

Navarro, J., \& Benz, W., 1991, ApJ, 380, 320

Nelson, A.F., 2000, ApJ, 537, L65

Nelson, A.F., 2003, in ASP Conf. Ser. 294, Scientific Frontiers in Research on Extrasolar Planets, ed. D.Deming (San Francusco:ASP), in press

Nelson, A.F., Benz, W., Adams, F.C., \& Arnett, D. 1998, ApJ, 502, 342

Nelson, A.F., Benz, W., \& Ruzmaikina, T.V. 2000, ApJ, 529, 357

Nelson, R.P, Papaloizou J.C.B., Masset, F. \& Kley, W., 2000, MNRAS, 318, 18

Papaloizou, J.C.B., 2002, MNRAS, A\&A, 388, 615 
Papaloizou, J.C.B., \& Larwood, J.D., 2000, MNRAS, 315, 823

Papaloizou, J.C.B., \& Terquem, C., 2001, MNRAS, 325, 221

Pickett, B.K., Cassen, P., Durisen, R.H., \& Link, R., 1998, ApJ, 504, 468

Pickett, B.K., Cassen, P., Durisen, R.H., \& Link, R. 2000a, ApJ, 529, 1034

Pickett, B.K., Durisen, R.H., Cassen, P., \& Mejia, A.C. 2000b, ApJ, 540, L95

Pickett, B.K., Meija, A., Durisen, R.H., Cassen, P.M., Berry, D.K.,\& Link, R.P., 2003, ApJ, 590,1060

Rice, W.K.M., Armitage, P.J., Bate, M.R., \& Bonnell, I.A., 2003, MNRAS, 339, 1025

Pollack, J.B., et al., 1996, Icarus, 124, 62

Romeo, A.B., 1992, MNRAS, 256, 307

Romeo, A.B., 1994, A\&A, 799, 806

Stadel. J., 2001, PhD thesis

Tanaka, H., Takeuchi, T., \& Ward, W. 2002, ApJ, 565, 1257

Terquem, C., \& Papaloizou, J.C.B., 2002, 332, L39

Thacker, R.J., Tittley, E.R., Pearce, F.R., Couchman, H.M.P., \& Thomas, P.A., 2000, MNRAS, 319, 619

Throop, H.B., Bally, J., Esposito,J.L.W., McCaughrean, M.J., 2001, Science, 292, 1686

Toomre, A., 1964, ApJ, 139, 1217

Udry, S., Mayor, M., Naef, D., Pepe, F., Queloz, D., Santos, N.C.,\& Burnet, M., 2002, A\&A 390, 267

Wadsley, J., Stadel, J., \& Quinn, T.R., New Astronomy, in press

Ward, W.R., 1997a, Icarus, 126, 261

Ward, W.R., 1997b, Icarus, L211-L214

Wetherill, G.W. 1990, Ann. Rev. Earth Planet. Sci., 18, 205

Weidenschilling, S.J., ApSS, 1977, 51, 153 
Yorke, H.W., \& Bodenheimer, P., ApJ, 1999, 525, 330 

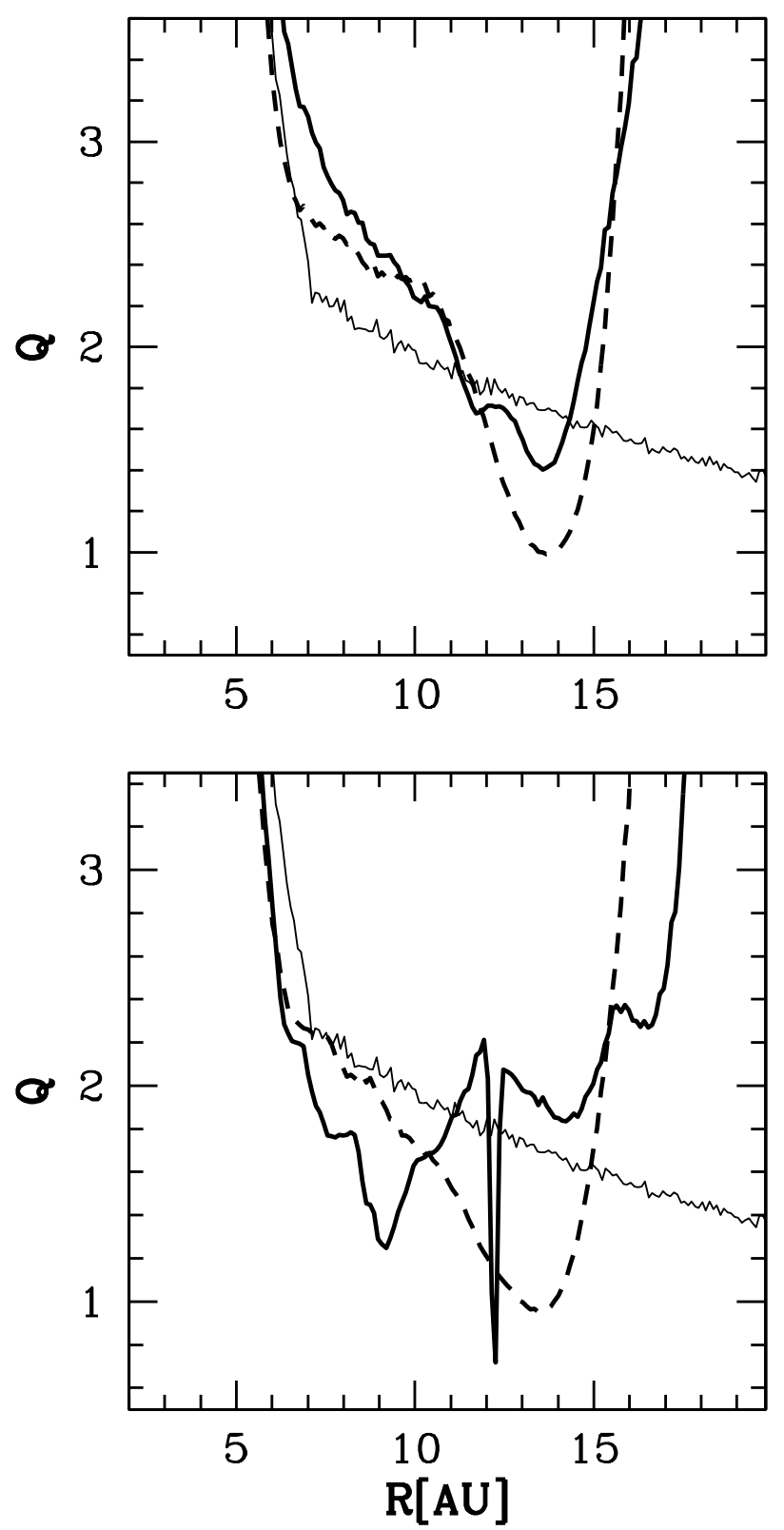

Fig. 1.- Evolution of Q profiles. Upper panel: model DISH2. Lower panel:model DISH1. We show the profiles at $\mathrm{t}=0$ (thin solid line), $\mathrm{t}=160$ years (dashed line) and $\mathrm{t}=240$ years (thick solid line). Fragmentation occurs between 160 years and 240 years in model DISH1, while model DISH2 develops only strong spiral arms. 


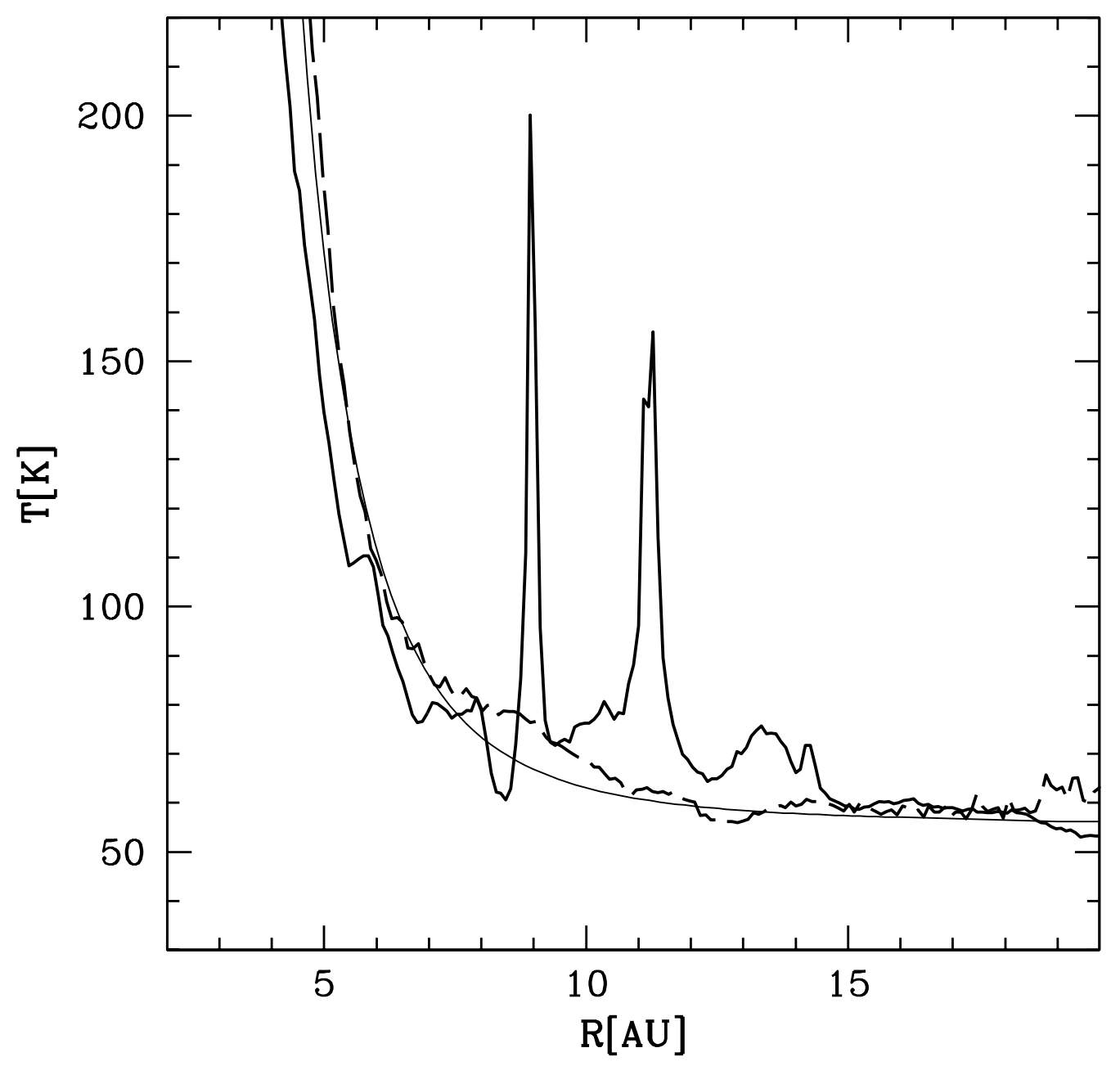

Fig. 2.- Evolution of the temperature profile. The initial profile (thin solid line) for an outer temperature of $56 \mathrm{~K}$ is shown - this profile was used for both model DISH1 and DISH2 (see Table 1). Model DISH1 undergoes clump formation and its profile is shown at $\mathrm{T}=320$ years (thick solid line), after the equation of state has been switched to adiabatic (see text). The peaks correspond to regions where bound clumps are (a single peak contains more than one clump due to limited bin size). Model DISH2 only forms spiral arms; its profile (thick dashed line) is also shown at $\mathrm{T}=320$ years (the equation of state, in this case, is locally isothermal throughout the evolution, so the small changes of the temperature with radius are due to radial diffusion of particles only). 

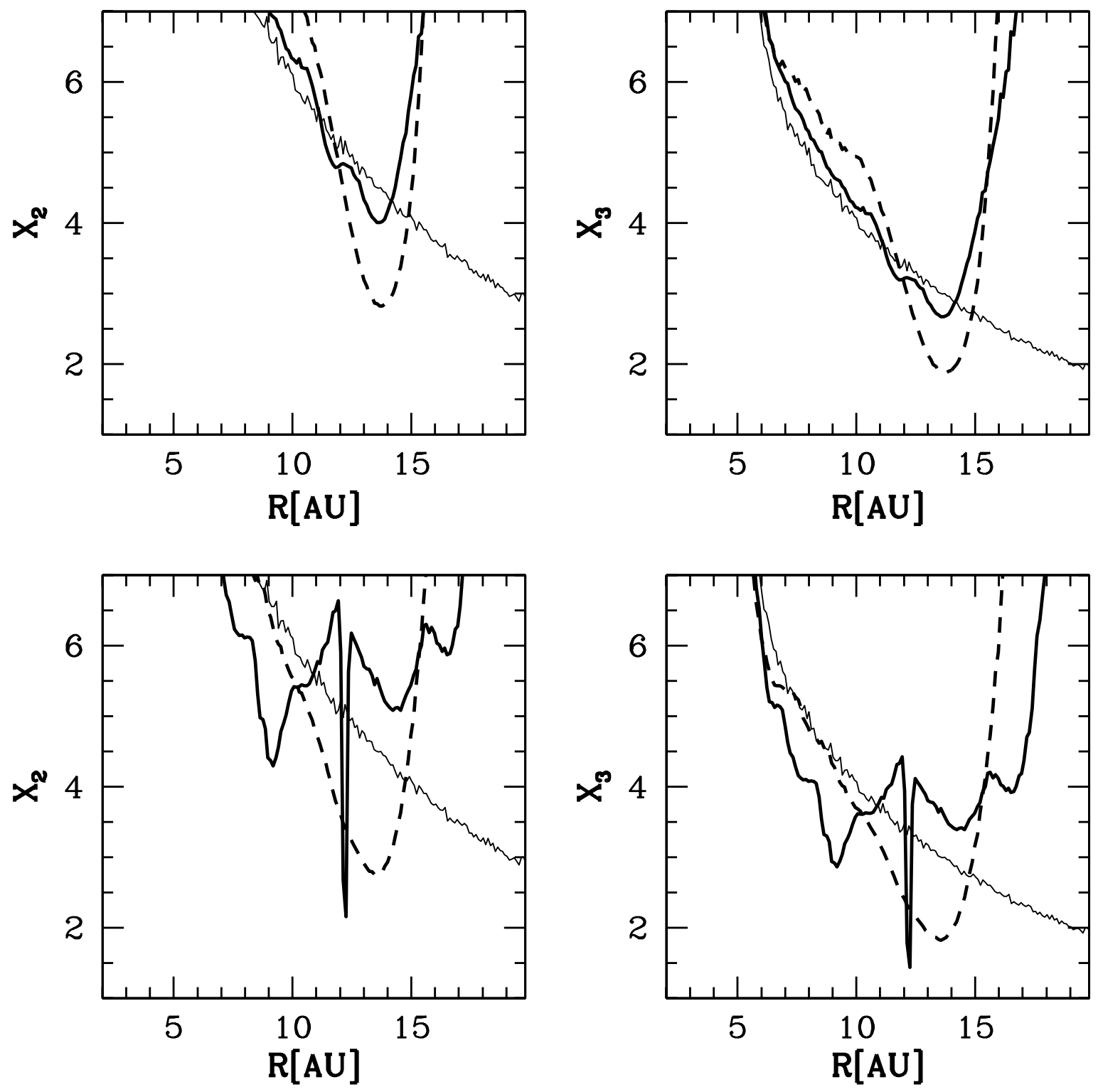

Fig. 3.- Evolution of $X_{2}$ (left) and $X_{3}$ (right) profiles. Upper panels: model DISH2. Lower panels:model DISH1. Profiles are shown at $\mathrm{t}=0$ (thin solid line), $\mathrm{t}=160$ years (dashed line) and $t=240$ years (thick solid line). See also Figure 1 on the $Q$ profiles for the same models. 


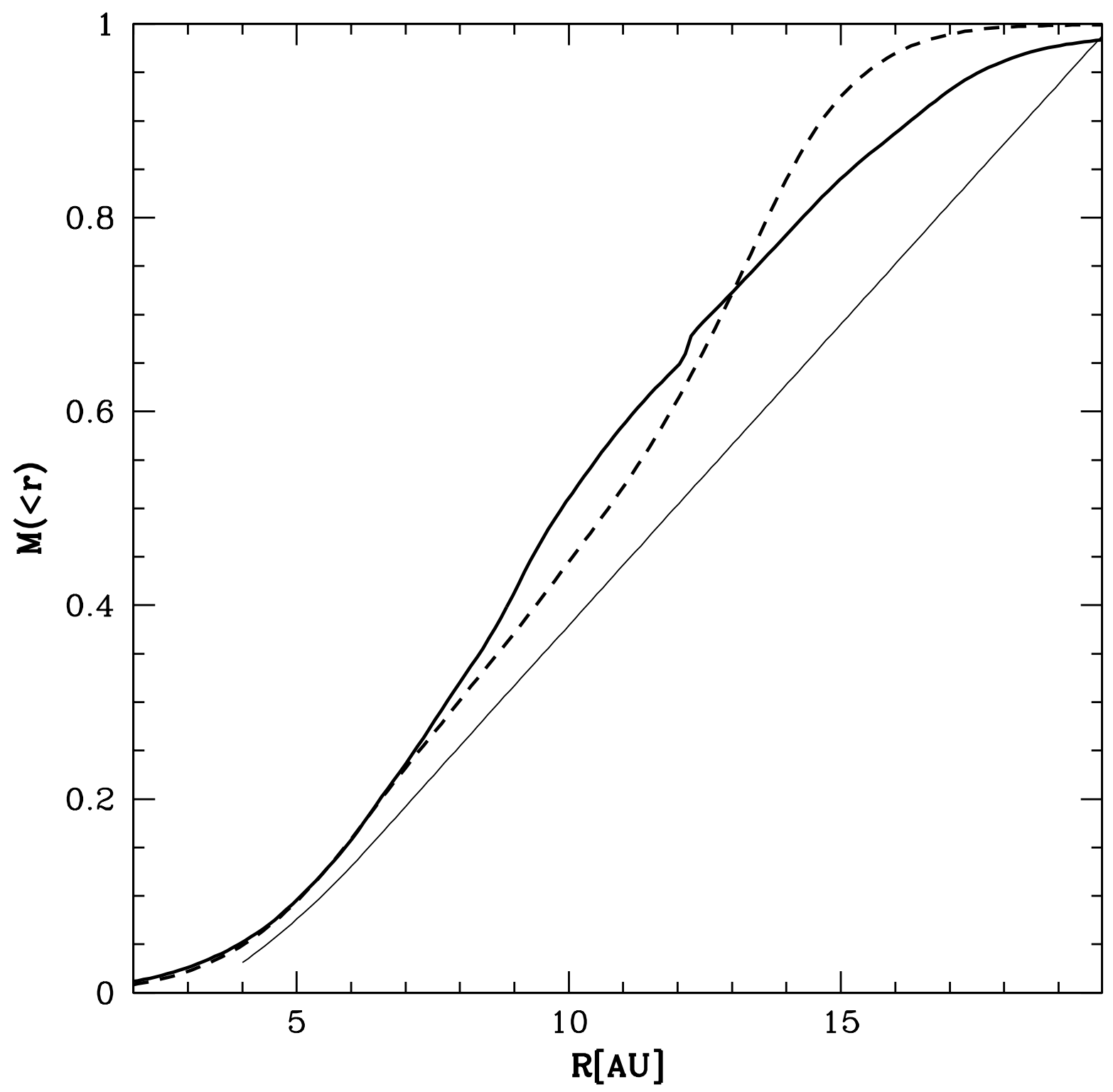

Fig. 4.- Evolution of the cumulative surface mass profile of model DISH1. The profile at $\mathrm{T}=0$ (thin solid line), at $\mathrm{T}=200$ years (dashed line - at this time the spiral arms have the strongest amplitude and are about to fragment) and at $\mathrm{T}=350$ years (thick solid line fragmentation has already occurred). 
Fig. 5.- Color-coded density maps of run DISH1 (see Table 1) after 350 years when the locally isothermal equation of state is used throughout the entire calculation (left panel) and when the simulation switches to an adiabatic equation of state (right panel) once the overdensities have grown past some threshold (see text, section 3.1) Brighter colors are for higher densities (densities between $10^{-14}$ and $10^{-6} \mathrm{~g} / \mathrm{cm}^{3}$ are shown using a logarithmic scale - the same applies to all density maps shown in this paper) and the disks are shown out to $20 \mathrm{AU}$.

Fig. 6.- Color-coded face-on density maps of run DISH3 (upper panels, $Q_{\min } \sim 1.3$ ) and DISH3b (lower panels, $Q_{\min } \sim 1.5$ ), at 200 (left) and 350 years (right). See Table 1 for details on the models. The equation of state is switched to adiabatic close to fragmentation in run DISH3. Brighter colors are for higher densities (see Figure 5) and the disks are shown out to $20 \mathrm{AU}$. 


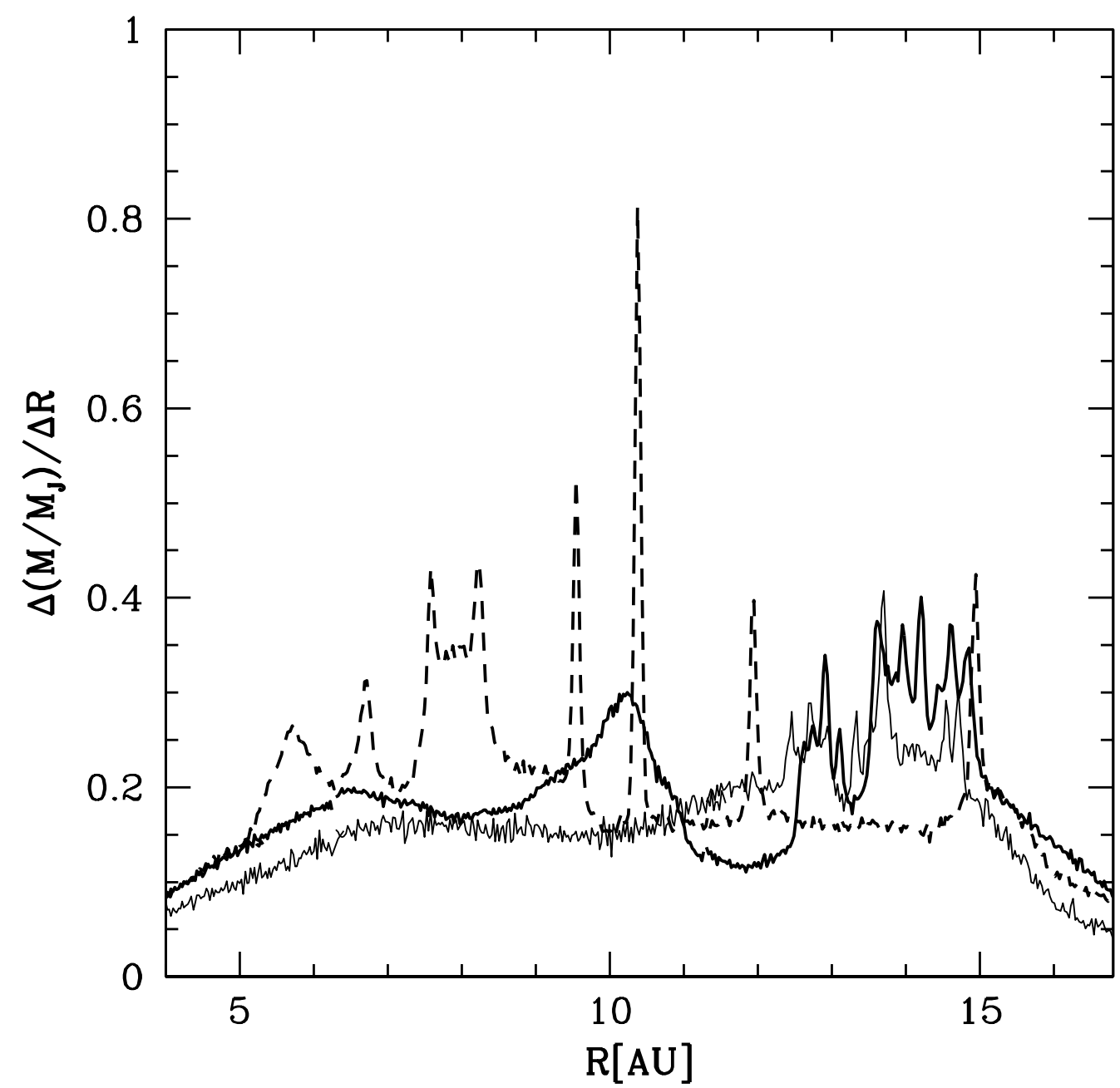

Fig. 7.- Radial mass profile at fragmentation. Results are shown for model DISH1 (dashed line), model DISH3 (thick solid line) and model DISgr (thin solid line). The mass is measured in units of one Jupiter mass, $M_{J}$, using cylindrical bins equally spaced in radius. The peaks correspond to bound clumps. Although the difference in the masses of the disks is small (e.g. DISH1 versus DISH3) the difference in the clumping mass scale is large because of the way Jeans length scales with mass and temperature (see section 3.1) 
Fig. 8.- Color-coded face-on density maps of run DISL4 after 120 (left panel) and 240 years (right panel), out to a radius if 25 AU. Note the stronger spiral arms compared to the runs shown in Figure 5, and the very eccentric orbital trail of the outermost clump in the right panel. 

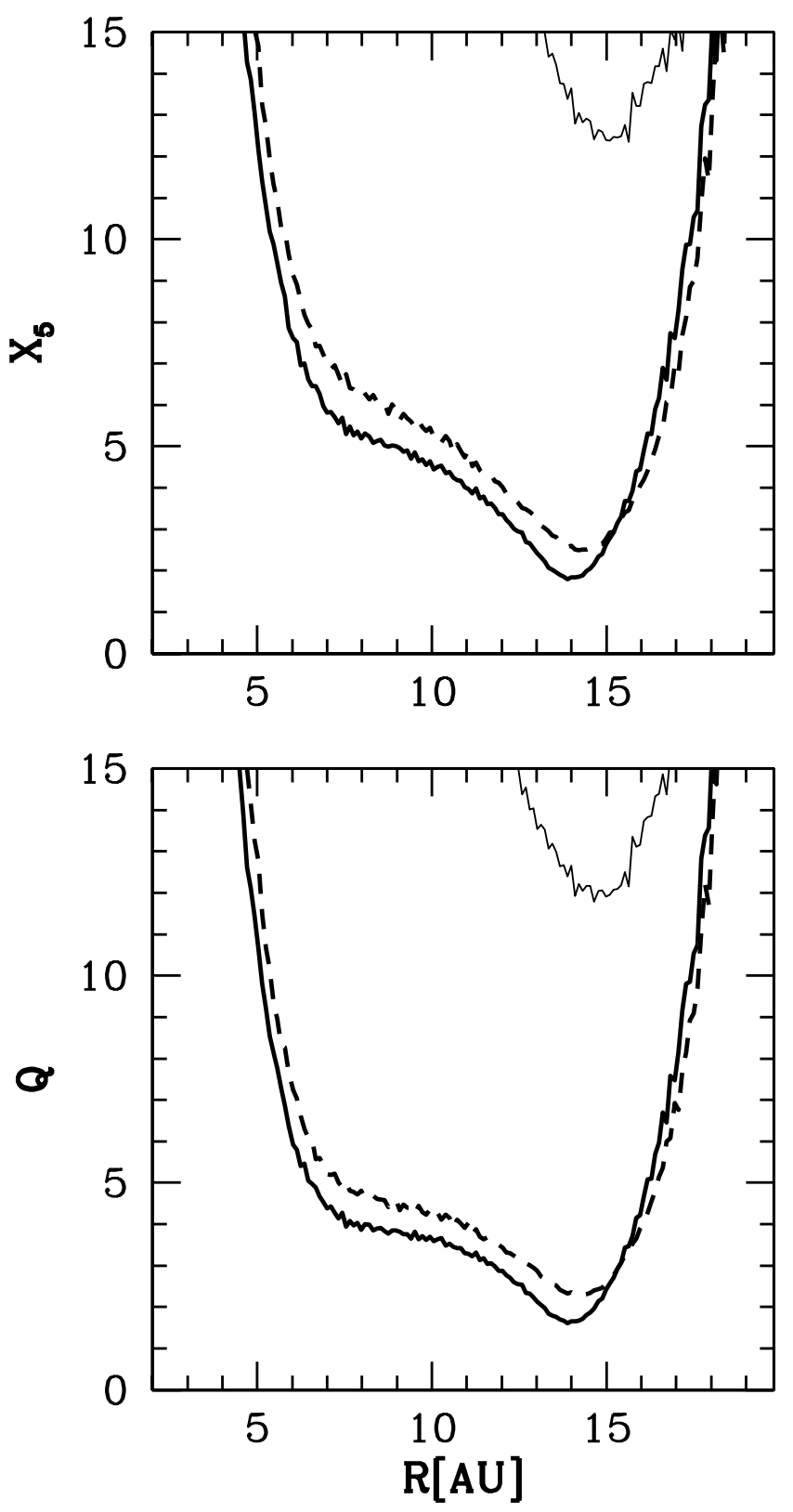

Fig. 9. - Evolution of the Toomre Q parameter (bottom) and of the $X_{5}$ parameter (top) for the growing disk in run DISgr (see Table and section 3.1.1.). The profiles is shown at 300 years (thin solid line), 450 years (dashed line) and 500 years (thick solid line - just before fragmentation starts. Note that the $X_{5}$ parameter is just below the threshold for instability $(<3)$ even at late times, which might explain why high order modes dominate in this run. 
Fig. 10.- Color-coded face-on density maps of adiabatic runs out to 20 AU. Brighter colors are used for higher densities (see Figure 1). The two upper panels show model DISLad3 at, respectively, 200 (left) and 300 (right) years, while the two lower panels show model DISLad4 at, at 200 (left) and 300 (right) years, respectively.

Fig. 11. - Color-coded face-on temperature maps of the adiabatic run DISLad4. Brighter colors are used for higher temperature (the scale goes from 20 to $2000 \mathrm{~K}$ ). Two snapshots are shown at, respectively, 120 years and 220 years (at this point clumps have just started forming), out to $20 \mathrm{AU}$ from the central star. Note the strong increase of temperature along the edges of the spiral arms and at the location of the clumps. 


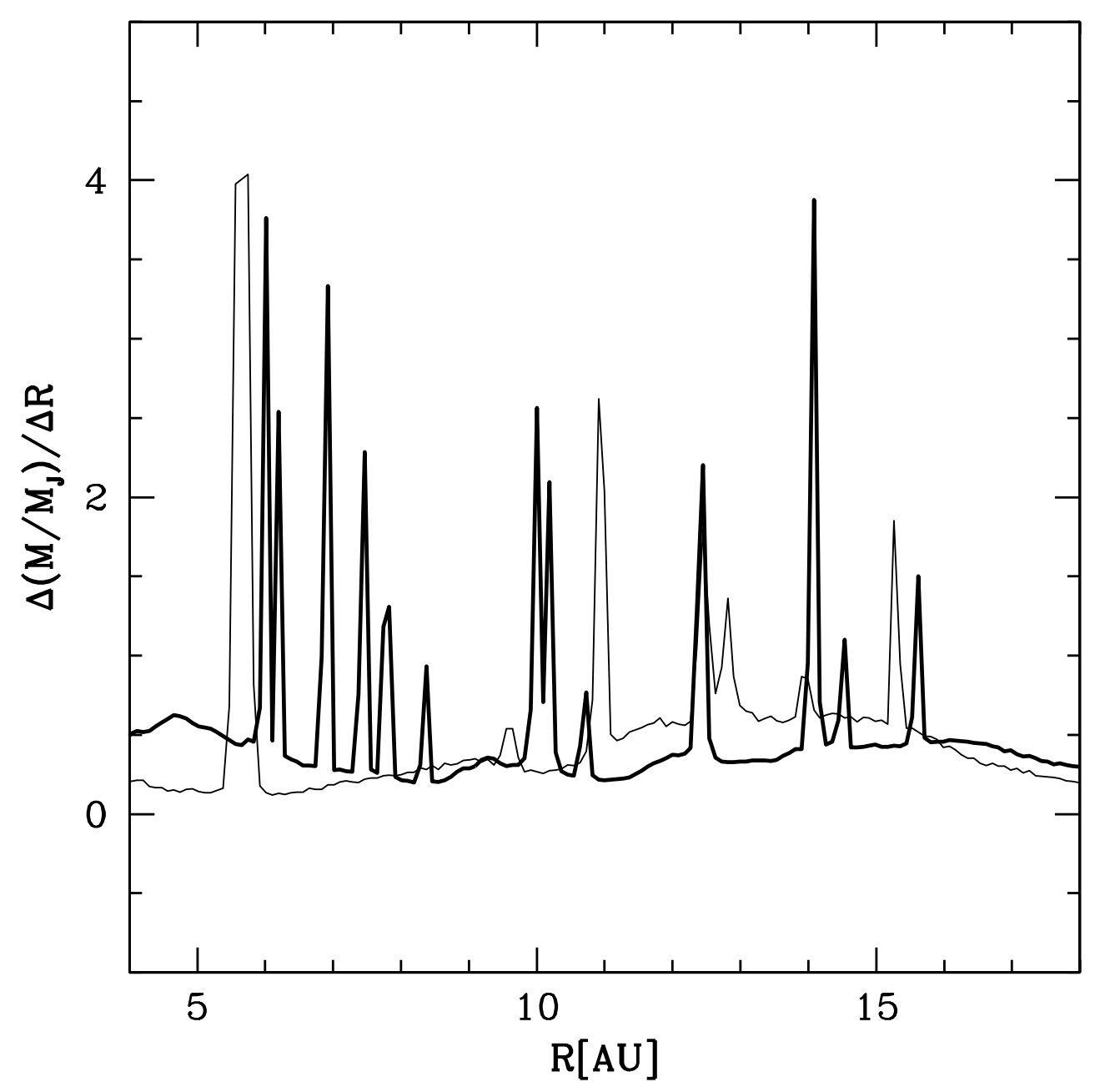

Fig. 12.- Differential mass profile after 350 years in two simulations with same disk model and different resolutions, run DISH1 (thick solid line) and run DISL1(thin solid line). The mass is measured in cylindrical radial bins, the unit is 1 Jupiter mass, $M_{J}$. See Table 1 for details on the simulations. Peaks correspond to gravitationally bound clumps. Clearly several more clumps are present in the higher resolution run. 

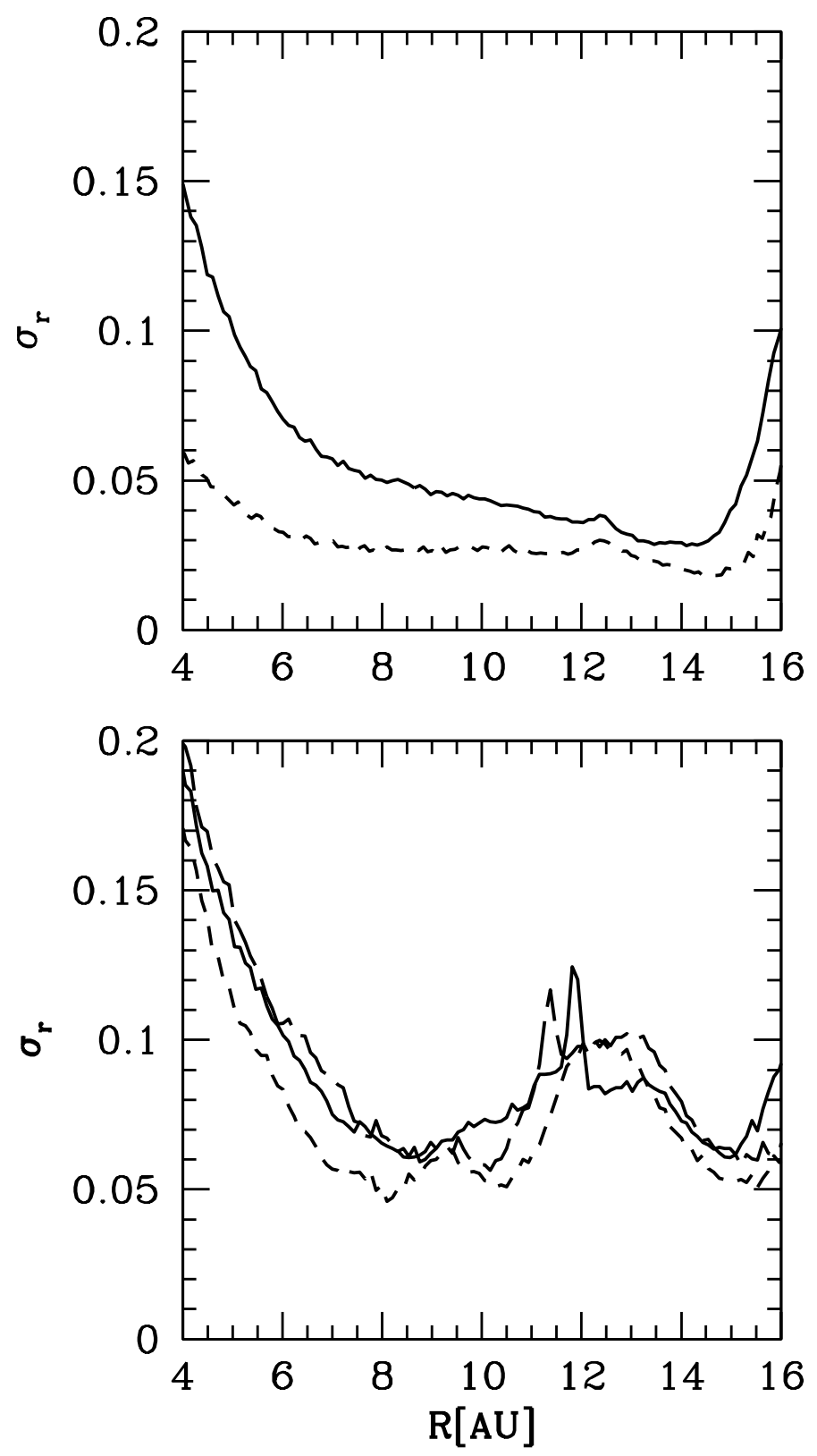

Fig. 13. - Effect of varying artificial viscosity on the velocity field of the disk in locally isothermal simulations. The radial velocity dispersion as a function of radius is shown. Regions where the radial dispersion is higher correspond to zones of higher non-axisymmetric motion or even collapse (the peaks). On top two weakly unstable models, DISH2 (solid line) and DISH2b (dashed line) are shown after 200 years of evolution; at the bottom three strongly unstable models, DISL1 (solid line), DISL1e (long-dashed line) and DISL1f (shortdashed line) are shown at the time of maximum growth of the spiral overdensities (just before fragmentation in the case of run DISL1, after about 160 years.) 
Fig. 14.- Color-coded density maps of the growing disk simulation, run DISgr. Brighter colors are for higher densities (see Figure 1) and the disk is shown out to 20 AU. From top to bottom and clockwise, snapshots are taken after 300, 480, 560 and 800 years.

Fig. 15.- Color-coded face-on density map of a run employing model DISL1 with a locally isothermal equation of state even after the appearance of the overdensities (see text, section 3.5). The box is $30 \mathrm{AU}$ on a side and the snapshot is taken after 450 years. Compared to the simulations employing adiabatic conditions once the overdensity threshold is reached (see Figure 14 and Figure 2 in Mayer et al. (2002)), it is evident that protoplanets are carving much clearer gaps, considerably more mass is piling up at the center and the disk is being dispersed much more quickly (the mean density is very low except in the central regions). 


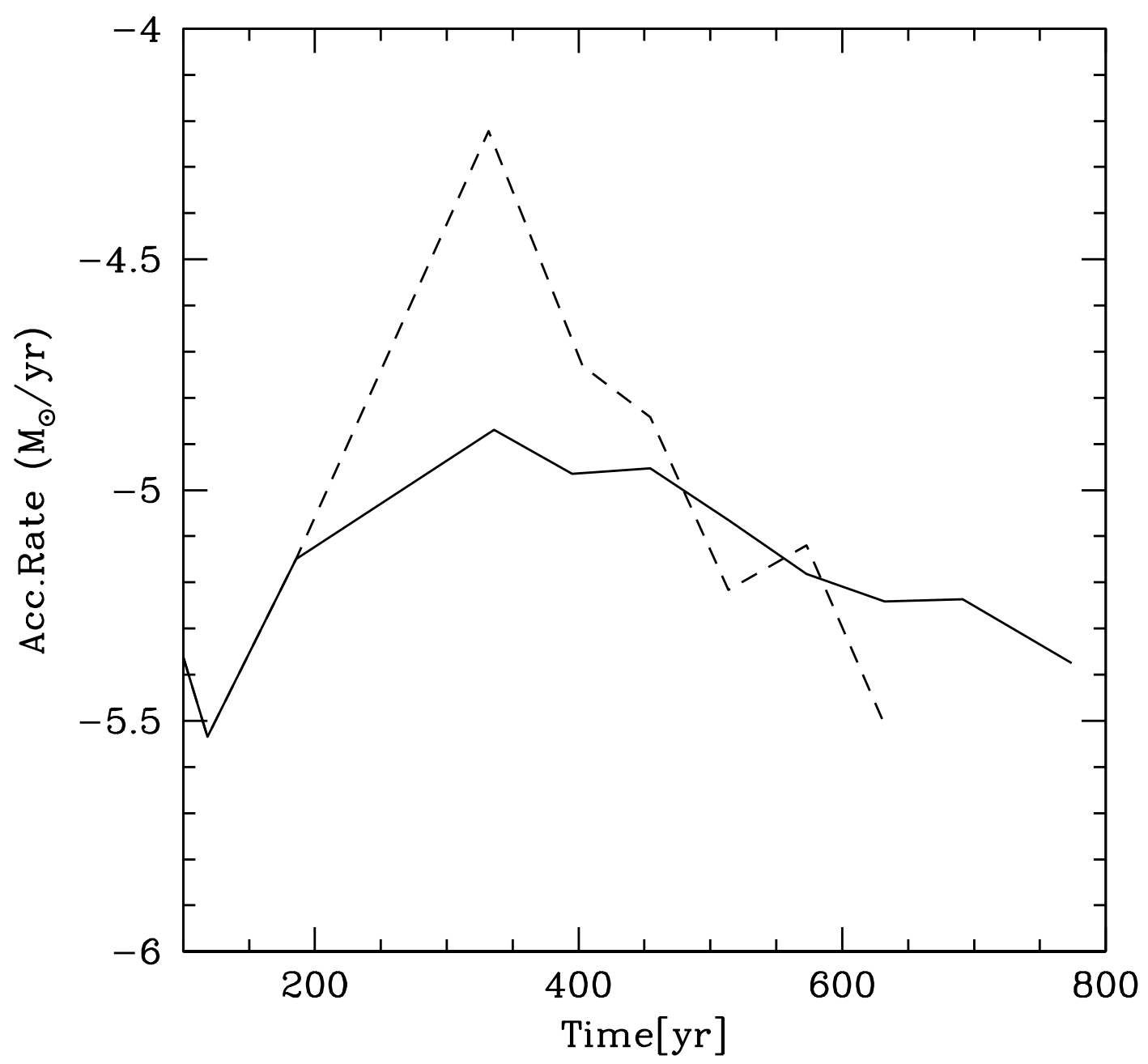

Fig. 16. - Accretion rate of gas onto the central star. The flow of mass inside a region of size equivalent to the gravitational softening length of the star ( $2 \mathrm{AU}$ ) is calculated. The simulations employ model DISL1 (see Table 1), the solid line is used for the case in which the equation of state is switched to adiabatic above the assigned density threshold, while the dashed line refers to the case in which the equation of state is locally isothermal for the entire duration of the calculation (see text, section 3.5). 


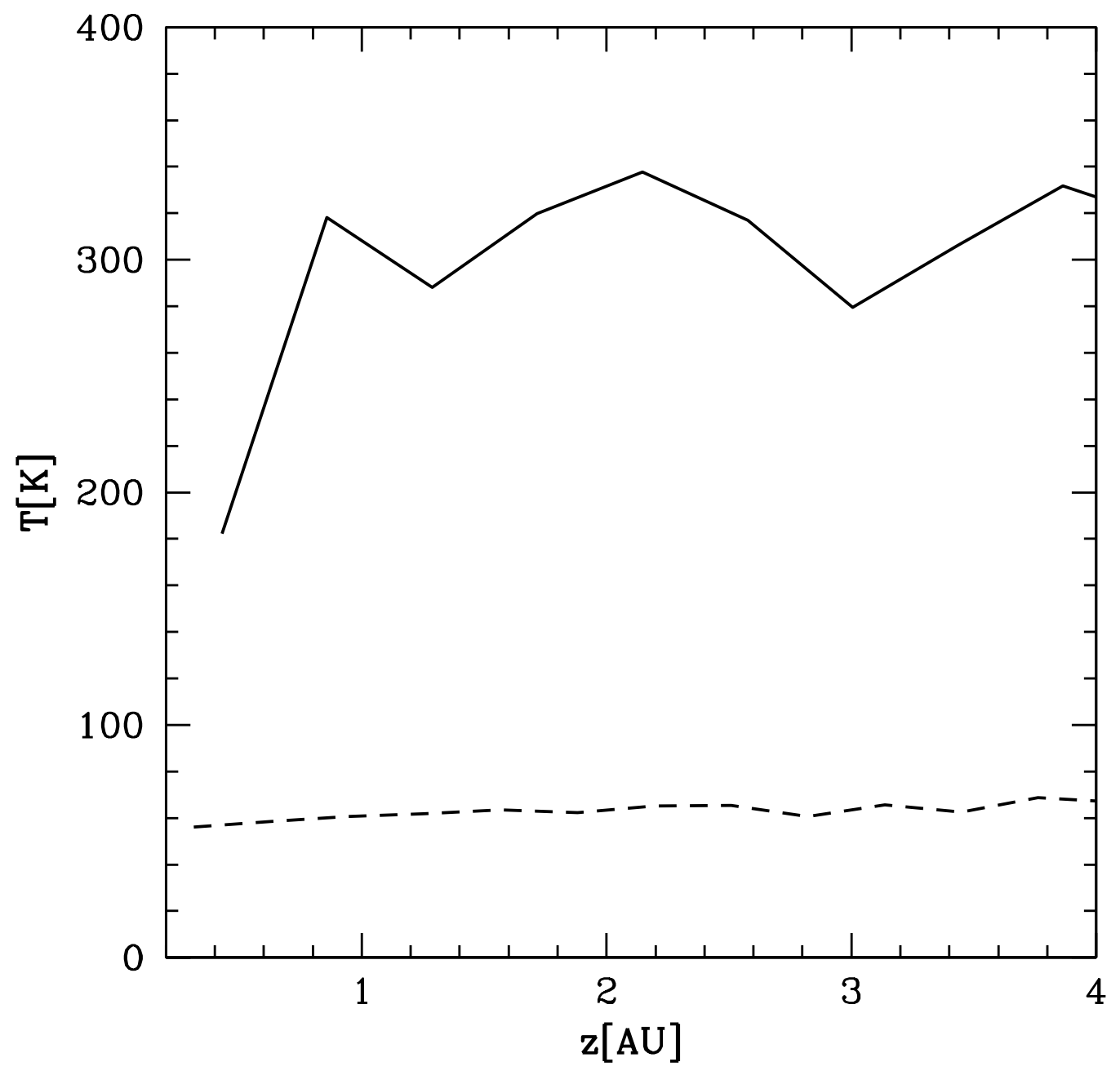

Fig. 17.- Temperature profile along the disk vertical axis for model DISL1 after 600 years for a fixed locally isothermal equation of state (dashed line) and for the case in which the equation of state is switched to adiabatic prior to clump formation (solid line). 
Fig. 18. - Close-up view of a gravitationally bound clump in run DISH1 (with the adiabatic switch) at 350 years. We show the projection perpendicular to the angular momentum axis of the clump. A color-coded plot of the velocity field is shown, the redder the color the higher the velocity. The clump is clearly in differential rotation. The box extends out to 0.5 AU and contains about 17574 particles. 
Table 1: Parameters of the simulations. Column 2: Initial disk mass as a fraction of stellar mass. Column 3: Equation of state (ISO for isothermal, otherwise $\gamma$ is indicated). Column 4: $\alpha$ parameter of artificial viscosity; Column 5: $\beta$ parameter in artificial viscosity. Column 7: number of disk particles Column 8: Softening of disk particles (in AU) Column 9: Mass of central star (in $M_{\odot}$ ); Column 10: Toomre Q parameter; Column 11: Outcome of the simulations in terms of clump formation ("yes" for formation of gravitationally bound clumps, "no" for no fragmentation at all, or "transient" for transient clumps).

\begin{tabular}{|c|c|c|c|c|c|c|c|c|c|c|}
\hline Run & $M_{d} / M_{s}$ & $E O S$ & $\alpha$ & $\beta$ & $N_{p}$ & $T_{\min }$ & softening & $M_{s}$ & $Q$ & clumps \\
\hline DISL1 & 0.1 & ISO & 1 & 2 & $2 \times 10^{5}$ & 56 & 0.12 & 1 & 1.38 & yes \\
\hline DISL1b & 0.1 & ISO & 1 & 2 & $2 \times 10^{5}$ & 56 & 0.18 & 1 & 1.38 & no \\
\hline DISL1c & 0.1 & ISO & 1 & 2 & $2 \times 10^{5}$ & 56 & 0.6 & 1 & 1.38 & no \\
\hline DISL1d & 0.1 & ISO & 1 & 2 & $2 \times 10^{5}$ & 56 & 0.06 & 1 & 1.38 & yes \\
\hline DISL1e & 0.1 & ISO & 1 & 2.5 & $2 \times 10^{5}$ & 56 & 0.12 & 1 & 1.38 & yes \\
\hline DISL1f & 0.1 & ISO & 1 & 3 & $2 \times 10^{5}$ & 56 & 0.12 & 1 & 1.38 & no \\
\hline DISL1g & 0.1 & ISO & 1 & 6 & $2 \times 10^{5}$ & 56 & 0.12 & 1 & 1.38 & no \\
\hline DISL1h & 0.1 & ISO & 1 & 0.5 & $2 \times 10^{5}$ & 56 & 0.12 & 1 & 1.38 & yes \\
\hline DISL1i & 0.1 & ISO & 0 & 0.5 & $2 \times 10^{5}$ & 56 & 0.12 & 1 & 1.38 & yes \\
\hline DISL2 & 0.1 & ISO & 1 & 2 & $2 \times 10^{5}$ & 100 & 0.06 & 1 & 2 & no \\
\hline DISH1 & 0.1 & ISO & 1 & 2 & $10^{6}$ & 56 & 0.06 & 1 & 1.38 & yes \\
\hline DISH2 & 0.08 & ISO & 1 & 2 & $10^{6}$ & 56 & 0.06 & 1 & 1.65 & no \\
\hline DISH2b & 0.08 & ISO & 0 & 0.5 & $10^{6}$ & 56 & 0.06 & 1 & 1.65 & no \\
\hline DISH2c & 0.08 & ISO & 0 & 0.5 & $10^{6}$ & 56 & 0.006 & 1 & 1.65 & yes \\
\hline DISH3 & 0.085 & ISO & 1 & 2 & $10^{6}$ & 36 & 0.06 & 1 & 1.3 & yes \\
\hline DISH3b & 0.085 & ISO & 1 & 2 & $10^{6}$ & 50 & 0.06 & 1 & 1.5 & no \\
\hline DISgr & 0.0085 & ISO & 1 & 2 & $2 \times 10^{5}$ & $30(\mathrm{gr})$ & 0.06 & 1 & $\mathrm{Q}(\mathrm{t})$ & yes \\
\hline DISH4 & 0.075 & ISO & 1 & 2 & $10^{6}$ & 56 & 0.06 & 1 & 1.9 & no \\
\hline DISH4b & 0.075 & ISO & 1 & 2 & $10^{6}$ & 56 & 0.006 & 1 & 1.9 & no \\
\hline DISL3 & 0.075 & ISO & 1 & 2 & $2 \times 10^{5}$ & 56 & 0.06 & 1 & 1.9 & no \\
\hline DISL4 & 0.075 & ISO & 1 & 2 & $2 \times 10^{5}$ & 56 & 0.06 & 0.5 & 1.38 & yes \\
\hline DISLad1 & 0.1 & 1.4 & 1 & 2 & $2 \times 10^{5}$ & 56 & 0.06 & 1 & 1.38 & no \\
\hline DISLad2 & 0.125 & 1.4 & 1 & 2 & $2 \times 10^{5}$ & 20 & 0.06 & 1 & 0.8 & no \\
\hline DISLad3 & 0.125 & 1.3 & 1 & 2 & $2 \times 10^{5}$ & 20 & 0.06 & 1 & 0.8 & transien \\
\hline DISLad4 & 0.125 & 1.2 & 1 & 2 & $2 \times 10^{5}$ & 20 & 0.06 & 1 & 0.8 & yes \\
\hline DISLad5 & 0.1 & 1.4 & 1 & 0.5 & $2 \times 10^{5}$ & 56 & 0.06 & 1 & 1.38 & no \\
\hline DISLad6 & 0.1 & 1.4 & 0 & 0.5 & $2 \times 10^{5}$ & 56 & 0.06 & 1 & 1.38 & no \\
\hline
\end{tabular}


This figure "f5b.gif" is available in "gif" format from: http://arxiv.org/ps/astro-ph/0310771v1 
This figure "f6b.gif" is available in "gif" format from: http://arxiv.org/ps/astro-ph/0310771v1 
This figure "f8b.gif" is available in "gif" format from: http://arxiv.org/ps/astro-ph/0310771v1 
This figure "f10b.gif" is available in "gif" format from: http://arxiv.org/ps/astro-ph/0310771v1 
This figure "f11b.gif" is available in "gif" format from: http://arxiv.org/ps/astro-ph/0310771v1 
This figure "f14b.gif" is available in "gif" format from: http://arxiv.org/ps/astro-ph/0310771v1 
This figure "f15b.gif" is available in "gif" format from: http://arxiv.org/ps/astro-ph/0310771v1 
This figure "f18b.gif" is available in "gif" format from: http://arxiv.org/ps/astro-ph/0310771v1 